\title{
Spin Three-Body Problem of Classical Electrodynamics with Radiation Terms - (I) Derivation of Spin Equations
}

\author{
Vasil G. Angelov \\ University of Mining and Geology "St. I. Rilski", Department of Mathematics, 1700 Sofia, Bulgaria.
}

\begin{abstract}
In the present paper spin equations for three-body problem of classical electrodynamics are introduced. They should be considered jointly with 3-body equations of motion derived in a previous paper of the author. The system of spin equations is an overdetermined one. It is shown that the independent spin equations are nine in number as many as the components of the unknown spin functions. The system obtained will be solved by the fixed-point method in a next paper.
\end{abstract}

Keywords: Spin three-body problem, Classical electrodynamics, Spin equations. 2010 MSC: 78A35.

\section{Introduction}

The present paper is one of the series devoted to study of the three-body problem of classical electrodynamics. In [5] we have formulated 3-dimensional three-body problem with radiation terms and in [6] we have proved the existence-uniqueness of periodic solution based on the previous results for two-body problem [2], [3. The system of equations of motion is of neutral type with delays depending on unknown trajectories. We have derived spin equations for two-body problem in [4] using the Corben's considerations of classical spinning particles [10], [11]. The problem for spinning particles in many papers has been considered (cf. for instance [7, [14], [16]-[20]). Here in a similar way we extend the results from [4] applying the interaction presentation for N-body problem from [1] and derive spin equations for three charged particles. We show that to every particle correspond six equations but three of them are consequences from the first three ones

Email address: angelov@mgu.bg ( Vasil G. Angelov) 
in the following sense: every such equation differs from the first one by a summand which is a cross product of spin vector and arbitrary constant vector perpendicular to the spin vector. So, we obtain nine equations for nine unknown spin functions, namely the component of the three spin vectors. We note the papers D.-A. Deckert et al. [8], [9], [12, [13], [15] based on the Wheeler- Feynman approach. The paper consists of five sections, Appendix and References. In Section 2 preliminary results for 3-body problem equations of motion with radiation terms and their relations to spin equations are considered. In Section 3 the spin equations are derived and the elements of the spin tensor are defined. In Section 4 a vector form of the Lorentz part and radiation parts of the first three spin equations is presented. Lorentz parts we call the summands in the right-hand sides of the spin equations which take into account for a given particle the influence of the rest ones. The terms which take into account self-interaction of every particle we call radiation terms. In Section 5 we show that the last three equations are consequences of the first three ones. In this way we obtain nine equations for nine unknown spin functions, or we get as many equations as spin functions are. The existence-uniqueness of a periodic solution of the general system in the second part of the present paper will be proved.

\section{Preliminary Results for3-Body Problem Equations of Motion with Radiation Terms and their Relations to Spin Equations}

First, we recall some denotations from [2] - [6]. The investigations are in the Minkowski space. Roman suffixes run over 1, 2, 3, 4, while Greek - over 1, 2, 3 with Einstein summation convention. By $\langle., .\rangle_{4}$ we denote the scalar product in the Minkowski space, while by $\langle.,$.$\rangle - the scalar product in three-dimensional$ Euclidean subspace.

Following the approach from [4] and [10] we introduce spin equations jointly with equations of motion with radiation terms derived in [5] for three-body problem:

$$
\begin{gathered}
\frac{d \lambda_{r}^{(1)}}{d s_{1}}=\frac{e_{1}}{m_{1} c^{2}}\left(\left(F_{r s}^{(12)}+F_{r s}^{(13)}\right) \lambda_{s}^{(1)}+F_{r s}^{(1) r a d} \lambda_{s}^{(1)}\right) ; \\
\frac{d \lambda_{r}^{(2)}}{d s_{2}}=\frac{e_{2}}{m_{2} c^{2}}\left(\left(F_{r s}^{(21)}+F_{r s}^{(23)}\right) \lambda_{s}^{(2)}+F_{r s}^{(2) r a d} \lambda_{s}^{(2)}\right) ; \\
\frac{d \lambda_{r}^{(3)}}{d s_{3}}=\frac{e_{3}}{m_{3} c^{2}}\left(\left(F_{r s}^{(31)}+F_{r s}^{(32)}\right) \lambda_{s}^{(3)}+F_{r s}^{(3) r a d} \lambda_{s}^{(3)}\right) ; \\
\frac{d \sigma_{i j}^{(1)}}{d s_{1}}=\frac{e_{1}}{m_{1} c^{2}}\left(\left(F_{i m}^{(12)}+F_{i m}^{(13)}+F_{i m}^{(1) r a d}\right) \sigma_{m j}^{(1)}-\sigma_{i m}^{(1)}\left(F_{m j}^{(12)}+F_{m j}^{(13)}+F_{m j}^{(1) r a d}\right)\right) ; \\
\frac{d \sigma_{i j}^{(2)}}{d s_{2}}=\frac{e_{2}}{m_{2} c^{2}}\left(\left(F_{i m}^{(21)}+F_{i m}^{(23)}+F_{i m}^{(2) r a d}\right) \sigma_{m j}^{(2)}-\sigma_{i m}^{(2)}\left(F_{m j}^{(21)}+F_{m j}^{(23)}+F_{m j}^{(2) r a d}\right)\right) ; \\
\frac{d \sigma_{i j}^{(3)}}{d s_{3}}=\frac{e_{3}}{m_{3} c^{2}}\left(\left(F_{i m}^{(31)}+F_{i m}^{(32)}+F_{i m}^{(3) r a d}\right) \sigma_{m j}^{(3)}-\sigma_{i m}^{(3)}\left(F_{m j}^{(31)}+F_{m j}^{(32)}+F_{m j}^{(3) r a d}\right)\right) .
\end{gathered}
$$

The electromagnetic tensors $F_{r s}^{(k n)}$ in the right-hand sides we call Lorentz parts. For each particle, they take into account the influence of other particles. The quantities relating to the particles are:

$\left(x_{1}^{(k)}(t), x_{2}^{(k)}(t), x_{3}^{(k)}(t), x_{4}^{(k)}=i c t\right) \equiv\left(\vec{x}^{(k)}, i c t\right),(k=1,2,3)$ - space-time coordinates of the moving particles; $c$ - the vacuum speed of light; $L_{k}$ - the world lines; $m_{k}$ - proper masses; $e_{k}$ - charges $(k=1,2,3)$. The elements of the electromagnetic tensors $F_{r l}^{(k n)}=\frac{\partial \Phi_{l}^{(n)}}{\partial x_{r}^{(k)}}-\frac{\partial \Phi_{r}^{(n)}}{\partial x_{l}^{(k)}}$ can be calculated by the retarded 
Lienard-Wiechert potentials $\Phi_{r}^{(n)}=-\frac{e_{n} \lambda_{r}^{(n)}}{\left\langle\lambda^{(n)}, \xi^{(k n)}\right\rangle_{4}}$, where

$$
\begin{aligned}
\xi^{(k n)}= & \left(\xi_{1}^{(k n)}, \xi_{2}^{(k n)}, \xi_{3}^{(k n)}, \xi_{4}^{(k n)}\right) \\
= & \left(x_{1}^{(k)}(t)-x_{1}^{(n)}\left(t-\tau_{k n}\right), x_{2}^{(k)}(t)-x_{2}^{(n)}\left(t-\tau_{k n}\right), x_{3}^{(k)}(t)-x_{3}^{(n)}\left(t-\tau_{k n}\right), i c \tau_{k n}(t)\right) ; \\
\lambda^{(k)}= & \left(\lambda_{1}^{(k)}, \lambda_{2}^{(k)}, \lambda_{3}^{(k)}, \lambda_{4}^{(k)}\right)=\left(\vec{\lambda}^{(k)}, \lambda_{4}^{(k)}\right)=\left(\frac{u_{1}^{(k)}}{\Delta_{k}}, \frac{u_{2}^{(k)}}{\Delta_{k}}, \frac{u_{3}^{(k)}}{\Delta_{k}}, \frac{i c}{\Delta_{k}}\right)=\left(\frac{\vec{u}^{(k)}}{\Delta_{k}}, \frac{i c}{\Delta_{k}}\right) ; \\
& \Delta_{k}=\sqrt{c^{2}-\left\langle\vec{u}^{(k)}(t), \vec{u}^{(k)}(t)\right\rangle} ; \Delta_{n}=\sqrt{c^{2}-\left\langle\vec{u}^{(n)}\left(t-\tau_{k n}\right), \vec{u}^{(n)}\left(t-\tau_{k n}\right)\right\rangle} .
\end{aligned}
$$

Since $\xi^{(k n)}$ are isotropic 4-vectors, i.e. $\left\langle\xi^{(k n)}, \xi^{(k n)}\right\rangle_{4}=0$, the retarded functions $\tau_{k n}(t)$ can be defined as solutions of the functional equations,

$$
\tau_{k n}(t)=\frac{1}{c} \sqrt{\left\langle\vec{\xi}^{(k n)}, \vec{\xi}^{(k n)}\right\rangle} \equiv \frac{1}{c} \sqrt{\sum_{\alpha=1}^{3}\left[x_{\alpha}^{(k)}(t)-x_{\alpha}^{(n)}\left(t-\tau_{k n}(t)\right]^{2}\right.},
$$

where $(k n)=(12),(13),(21),(23),(31),(32)$.

Following denotations from [5] we obtain

$$
\begin{aligned}
& \frac{d \lambda_{r}^{(k)}}{d s_{k}}=\frac{e_{k}}{m_{k} c^{2}} \sum_{n=1, n \neq k}^{3} F_{r m}^{(k n)} \lambda_{m}^{(k)} ; F_{r m}^{(k n)}=\frac{\partial \Phi_{m}^{(n)}}{\partial x_{r}^{(k)}}-\frac{\partial \Phi_{r}^{(n)}}{\partial x_{m}^{(k)}}=e_{n}\left(P_{r}^{(k n)} \xi_{m}^{(k n)}-P_{m}^{(k n)} \xi_{r}^{(k n)}\right) ; \\
& \frac{d \lambda_{r}^{(k)}}{d s_{k}}=\frac{e_{k}}{m_{k} c^{2}} \sum_{n=1, n \neq k}^{3} e_{n}\left(P_{r}^{(k n)} \xi_{m}^{(k n)}-P_{m}^{(k n)} \xi_{r}^{(k n)}\right) \lambda_{m}^{(k)} \\
& \frac{d \vec{\lambda}^{(k)}}{d s_{k}}=-\frac{e_{k}^{2}}{m_{k} c^{2}} \sum_{n=1, n \neq k}^{3} e_{n}\left[\vec{P}^{(k n)}\left\langle\lambda^{(k)}, \xi^{(k n)}\right\rangle_{4}-\vec{\xi}^{(k n)}\left\langle\lambda^{(k)}, P^{(k n)}\right\rangle_{4}\right] \\
& \frac{d \vec{\lambda}^{(k)}}{d s_{k}}=\frac{1}{\Delta_{k}^{2}} \frac{d \vec{u}^{(k)}(t)}{d t}+\frac{\vec{u}^{(k)}(t)}{\Delta_{k}^{4}}\left\langle\vec{u}^{(k)}(t), \frac{d \vec{u}^{(k)}(t)}{d t}\right\rangle ; \frac{d \lambda_{4}^{(k)}}{d s_{k}}=\frac{i c}{\Delta_{k}^{4}}\left\langle\vec{u}^{(k)}(t), \frac{d \vec{u}^{(k)}(t)}{d t}\right\rangle ; \\
& D_{k n}=\frac{c^{2} \tau_{k n}-\left\langle\vec{\xi}^{(k n)}, \vec{u}^{(n)}\right\rangle}{c^{2} \tau_{k n}-\left\langle\vec{\xi}^{(k n)}, \vec{u}^{(k)}\right\rangle} ; \frac{d \vec{\lambda}^{(n)}}{d s_{n}}=\frac{D_{k n}}{\Delta_{n}^{2}}\left(\frac{d \vec{u}^{(n)}}{d t}+\frac{\vec{u}^{(n)}}{\Delta_{k n}^{2}}\left\langle\vec{u}^{(n)}, \frac{d \vec{u}^{(n)}}{d t}\right\rangle\right) ; \\
& \frac{d \lambda_{4}^{(n)}}{d s_{n}}=\frac{i c D_{k n}}{\Delta_{k n}^{4}}\left\langle\vec{u}^{(n)}, \frac{d \vec{u}^{(n)}}{d t}\right\rangle ; P_{r}^{(k n)}=-\frac{\lambda_{r}^{(n)}}{\left\langle\lambda^{(n)}, \xi^{(k n)}\right\rangle_{4}^{3}}\left[1+\left\langle\xi^{(k n)}, \frac{d \lambda^{(n)}}{d s_{n}}\right\rangle_{4}\right]+\frac{1}{\left\langle\lambda^{(n)}, \xi^{(k n)}\right\rangle_{4}{ }^{2}} \frac{d \lambda_{r}^{(n)}}{d s_{n}} ; \\
& \vec{P}^{(k n)}=-\frac{\vec{\lambda}^{(n)} M_{k n}}{\left\langle\lambda^{(n)}, \xi^{(k n)}\right\rangle_{4}^{3}}+\frac{1}{\left\langle\lambda^{(n)}, \xi^{(k n)}\right\rangle_{4}{ }^{2}} \frac{d \vec{\lambda}^{(n)}}{d s_{n}} ; M_{k n}=1+\left\langle\xi^{(k n)}, \frac{d \lambda^{(n)}}{d s_{n}}\right\rangle_{4} ; \\
& P_{4}^{(k n)}=-\frac{\lambda_{4}^{(n)} M_{k n}}{\left\langle\lambda^{(n)}, \xi^{(k n)}\right\rangle_{4}^{3}}+\frac{1}{\left\langle\lambda^{(n)}, \xi^{(k n)}\right\rangle_{4}{ }^{2}} \frac{d \lambda_{4}^{(n)}}{d s_{n}}=-i c\left(\frac{M_{k n}}{\Delta_{n}\left\langle\lambda^{(n)}, \xi^{(k n)}\right\rangle_{4}^{3}}-\frac{D_{k n}\left\langle\vec{u}^{(n)}, \dot{\vec{u}}^{(n)}\right\rangle}{\left\langle\lambda^{(n)}, \xi^{(k n)}\right\rangle_{4}{ }^{2} \Delta_{n}^{4}}\right) \equiv-i c L_{k n} ; \\
& \frac{i}{c} P_{4}^{(k n)}=L_{k n}(k=1,2,3 ; r=1,2,3,4) .
\end{aligned}
$$

The radiation terms are defined as a half of the difference of retarded and advanced potentials in accordance with the Dirac assumption:

$$
F_{m l}^{(k) r a d}=\frac{1}{2}\left[\left(\frac{\partial \Phi_{l}^{(k) r e t}}{\partial x_{m}^{(k) r e t}}-\frac{\partial \Phi_{m}^{(n) r e t}}{\partial x_{l}^{(k) r e t}}\right)-\left(\frac{\partial \Phi_{l}^{(k) a d v}}{\partial x_{m}^{(k) a d v}}-\frac{\partial \Phi_{m}^{(n) a d v}}{\partial x_{l}^{(k) a d v}}\right)\right],
$$


where $\Phi_{r}^{(k) r e t}=-\frac{e_{k} \lambda_{r}^{(k) r e t}}{\left\langle\lambda^{(k) r e t}, \xi^{(k) r e t}\right\rangle_{4}}, \Phi_{r}^{(k) a d v}=-\frac{e_{k} \lambda_{r}^{(k) a d v}}{\left\langle\lambda^{(k) a d v}, \xi^{(k) a d v}\right\rangle_{4}}$ and

$$
\begin{gathered}
F_{r s}^{(k) r e t}=\frac{\partial \Phi_{s}^{(k) r e t}}{\partial x_{r}^{(k)}}-\frac{\partial \Phi_{r}^{(k) r e t}}{\partial x_{s}^{(k)}}=e_{k}\left(P_{r}^{(k) r e t} \xi_{s}^{(k) r e t}-P_{s}^{(k) r e t} \xi_{r}^{(k) r e t}\right) \\
F_{r s}^{(k) a d v}=\frac{\partial \Phi_{s}^{(k) a d v}}{\partial x_{r}^{(k)}}-\frac{\partial \Phi_{r}^{(k) a d v}}{\partial x_{s}^{(k)}}=e_{k}\left(P_{r}^{(k) a d v} \xi_{s}^{(k) a d v}-P_{s}^{(k) a d v} \xi_{r}^{(k) a d v}\right),
\end{gathered}
$$

where

$$
\begin{gathered}
P_{r}^{(k) r e t}=-\frac{\lambda_{r}^{(k) r e t}}{\left\langle\lambda^{(k) r e t}, \xi^{(k) r e t}\right\rangle_{4}^{3}}\left[1+\left\langle\xi^{(k) r e t}, \frac{d \lambda^{(k) r e t}}{d s_{k}}\right\rangle_{4}\right]+\frac{1}{\left\langle\lambda^{(k) r e t}, \xi^{(k) r e t}\right\rangle_{4}{ }^{2}} \frac{d \lambda_{r}^{(k) r e t}}{d s_{k}} ; \\
P_{r}^{(k) a d v}=-\frac{\lambda_{r}^{(k) a d v}}{\left\langle\lambda^{(k) a d v}, \xi^{(k) a d v}\right\rangle_{4}^{3}}\left[1+\left\langle\xi^{(k) a d v}, \frac{d \lambda^{(k) a d v}}{d s_{k}}\right\rangle_{4}\right]+\frac{1}{\left\langle\lambda^{(k) a d v}, \xi^{(k) a d v}\right\rangle_{4}{ }^{2}} \frac{d \lambda_{r}^{(k) a d v}}{d s_{k}} ; \\
F_{r s}^{(k) r a d}=\frac{F_{r s}^{(k) r e t}-F_{r s}^{(k) a d v}}{2}=e_{k} \frac{P_{r}^{(k) r e t} \xi_{s}^{(k) r e t}-P_{s}^{(k) r e t} \xi_{r}^{(k) r e t}-\left(P_{r}^{(k) a d v} \xi_{s}^{(k) a d v}-P_{s}^{(k) a d v} \xi_{r}^{(k) a d v}\right)}{2} .
\end{gathered}
$$

In the previous paper [5] it is proved that every fourth equation of $(2.1)$ is a consequence of the first three ones. So under the assumption $\sqrt{\left\langle\vec{u}^{(k)}(t), \vec{u}^{(k)}(t)\right\rangle} \leq \bar{c}<c(k=1,2,3)$ the equations of motion 2.1 are 12 in number but it is proved that three of them are consequence of the rest ones so we obtain nine in number independent equations. In [6] an existence-uniqueness of a periodic solution of $[2.1]$ is proved.

\section{Derivation of the Spin Equations}

Recalling the summation in repeated Latin indices we introduce an explicit form of the spin equations:

$$
\begin{aligned}
& \frac{d \sigma_{i j}^{(1)}}{d s_{1}}=\frac{e_{1}}{m_{1} c^{2}}\left[\left(F_{i m}^{(12)}+F_{i m}^{(13)}+F_{i m}^{(1) r a d}\right) \sigma_{m j}^{(1)}-\sigma_{i m}^{(1)}\left(F_{m j}^{(12)}+F_{m j}^{(13)}+F_{m j}^{(1) r a d}\right)\right] ; \\
& \frac{d \sigma_{i j}^{(2)}}{d s_{2}}=\frac{e_{2}}{m_{2} c^{2}}\left[\left(F_{i m}^{(21)}+F_{i m}^{(23)}+F_{i m}^{(2) r a d}\right) \sigma_{m j}^{(2)}-\sigma_{i m}^{(2)}\left(F_{m j}^{(21)}+F_{m j}^{(23)}+F_{m j}^{(2) r a d}\right)\right] ; \\
& \frac{d \sigma_{i j}^{(3)}}{d s_{3}}=\frac{e_{3}}{m_{3} c^{2}}\left[\left(F_{i m}^{(31)}+F_{i m}^{(32)}+F_{i m}^{(3) r a d}\right) \sigma_{m j}^{(3)}-\sigma_{i m}^{(3)}\left(F_{m j}^{(31)}+F_{m j}^{(32)}+F_{m j}^{(3) r a d}\right)\right]
\end{aligned}
$$

or in abbreviated form

$$
\frac{d \sigma_{i j}^{(k)}}{d s_{k}}=\frac{e_{1}}{m_{1} c^{2}}\left[\left(\sum_{n=1, n \neq k}^{3} F_{i m}^{(k n)}+F_{i m}^{(k) r a d}\right) \sigma_{m j}^{(1)}-\sigma_{i m}^{(1)}\left(\sum_{n=1, n \neq k}^{3} F_{m j}^{(k n)}+F_{m j}^{(1) r a d}\right)\right] .
$$

Introduce denotations following [4]:

$$
\begin{aligned}
& \vec{\theta}^{(k)}=\left(\theta_{1}^{(k)}(t), \theta_{2}^{(k)}(t), \theta_{3}^{(k)}(t)\right) ; \vec{\sigma}^{(k)}=\left(\sigma_{1}^{(k)}, \sigma_{2}^{(k)}, \sigma_{3}^{(k)}\right) ; \\
& \vec{\theta}^{(k)}=\frac{1}{c}\left(\vec{\lambda}^{(k)} \times \vec{\sigma}^{(k)}\right)=\frac{1}{c}\left|\begin{array}{ccc}
\vec{e}_{1} & \vec{e}_{2} & \vec{e}_{3} \\
\lambda_{1}^{(k)} & \lambda_{2}^{(k)} & \lambda_{3}^{(k)} \\
\sigma_{1}^{(k)} & \sigma_{2}^{(k)} & \sigma_{3}^{(k)}
\end{array}\right|=\frac{1}{c}\left|\begin{array}{cc}
\lambda_{2}^{(k)} & \lambda_{3}^{(k)} \\
\sigma_{2}^{(k)} & \sigma_{3}^{(k)}
\end{array}\right| \vec{e}_{1}-\frac{1}{c}\left|\begin{array}{cc}
\lambda_{1}^{(k)} & \lambda_{3}^{(k)} \\
\sigma_{1}^{(k)} & \sigma_{3}^{(k)}
\end{array}\right| \vec{e}_{2}+\frac{1}{c}\left|\begin{array}{cc}
\lambda_{1}^{(k)} & \lambda_{2}^{(k)} \\
\sigma_{1}^{(k)} & \sigma_{2}^{(k)}
\end{array}\right| \vec{e}_{3}
\end{aligned}
$$

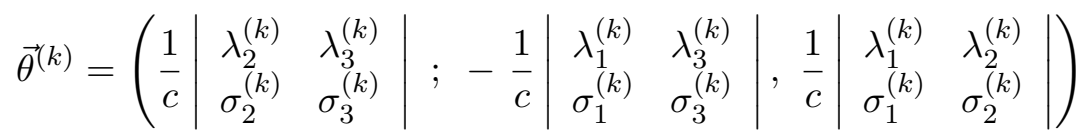

that is, 
we define the spin tensor

$$
\begin{aligned}
& \sigma_{\mu \nu}^{(k)}=\left(\begin{array}{cccc}
0 & \sigma_{3}^{(k)} & -\sigma_{2}^{(k)} & i \theta_{1}^{(k)} \\
-\sigma_{3}^{(k)} & 0 & \sigma_{1}^{(k)} & i \theta_{2}^{(k)} \\
\sigma_{2}^{(k)} & -\sigma_{1}^{(k)} & 0 & i \theta_{3}^{(k)} \\
-i \theta_{1}^{(k)} & -i \theta_{2}^{(k)} & -i \theta_{3}^{(k)} & 0
\end{array}\right)
\end{aligned}
$$

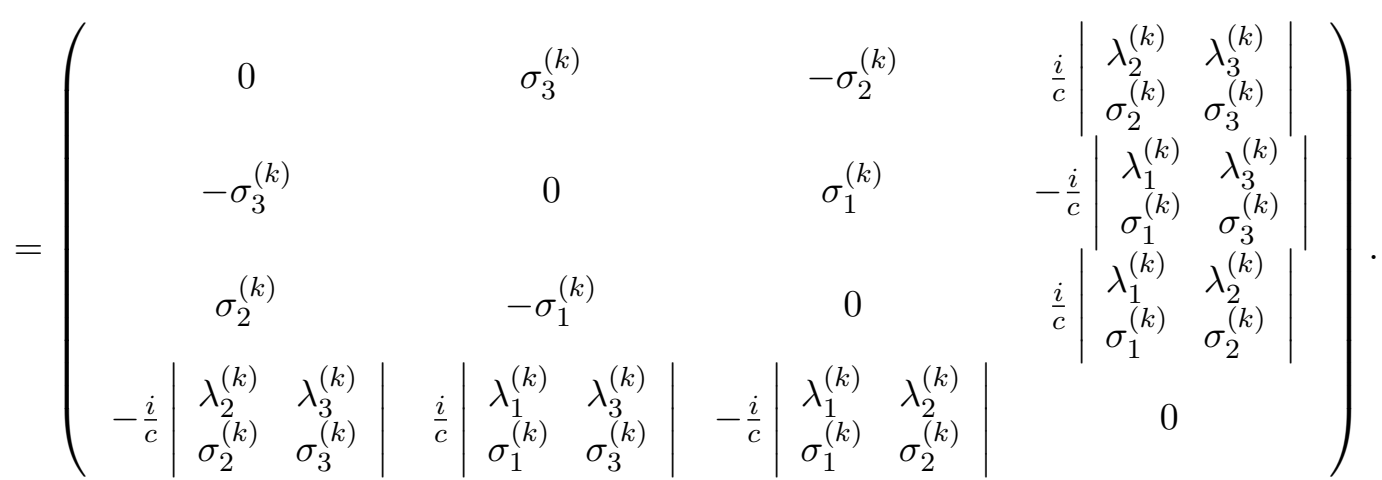

Thus, we introduce the following equations describing the spin of three charged particles:

or

$$
\begin{aligned}
& \frac{d \sigma_{12}^{(k)}}{d s_{k}}=\frac{e_{k}}{m_{k} c^{2}}\left[\left(\sum_{n=1, n \neq k}^{3} F_{1 m}^{(k n)}+F_{1 m}^{(k) r a d}\right) \sigma_{m 2}^{(k)}-\sigma_{1 m}^{(k)}\left(\sum_{n=1, n \neq k}^{3} F_{m 2}^{(k n)}+F_{m 2}^{(k) r a d}\right)\right] ; \\
& \frac{d \sigma_{13}^{(k)}}{d s_{k}}=\frac{e_{k}}{m_{k} c^{2}}\left[\left(\sum_{n=1, n \neq k}^{3} F_{1 m}^{(k n)}+F_{1 m}^{(k) r a d}\right) \sigma_{m 3}^{(k)}-\sigma_{1 m}^{(k)}\left(\sum_{n=1, n \neq k}^{3} F_{m 3}^{(k n)}+F_{m 3}^{(k) r a d}\right)\right] ; \\
& \frac{d \sigma_{23}^{(k)}}{d s_{k}}=\frac{e_{k}}{m_{k} c^{2}}\left[\left(\sum_{n=1, n \neq k}^{3} F_{2 m}^{(k n)}+F_{2 m}^{(k) r a d}\right) \sigma_{m 3}^{(k)}-\sigma_{2 m}^{(k)}\left(\sum_{n=1, n \neq k}^{3} F_{m 3}^{(k n)}+F_{m 3}^{(k) r a d}\right)\right] ; \\
& \frac{d \sigma_{14}^{(k)}}{d s_{k}}=\frac{e_{k}}{m_{k} c^{2}}\left[\left(\sum_{n=1, n \neq k}^{3} F_{1 m}^{(k n)}+F_{1 m}^{(k) r a d}\right) \sigma_{m 4}^{(k)}-\sigma_{1 m}^{(k)}\left(\sum_{n=1, n \neq k}^{3} F_{m 4}^{(k n)}+F_{m 4}^{(k) r a d}\right)\right] ; \\
& \frac{d \sigma_{24}^{(k)}}{d s_{k}}=\frac{e_{k}}{m_{k} c^{2}}\left[\left(\sum_{n=1, n \neq k}^{3} F_{2 m}^{(k n)}+F_{2 m}^{(k) r a d}\right) \sigma_{m 4}^{(k)}-\sigma_{2 m}^{(k)}\left(\sum_{n=1, n \neq k}^{3} F_{m 4}^{(k n)}+F_{m 4}^{(k) r a d}\right)\right] ; \\
& \frac{d \sigma_{34}^{(k)}}{d s_{k}}=\frac{e_{k}}{m_{k} c^{2}}\left[\left(\sum_{n=1, n \neq k}^{3} F_{3 m}^{(k n)}+F_{3 m}^{(k) r a d}\right) \sigma_{m 4}^{(k)}-\sigma_{3 m}^{(k)}\left(\sum_{n=1, n \neq k}^{3} F_{m 4}^{(k n)}+F_{m 4}^{(k) r a d}\right)\right]
\end{aligned}
$$

$\frac{d \sigma_{12}^{(k)}}{d s_{k}}=\frac{e_{k}}{m_{k} c^{2}} \sum_{n=1, n \neq k}^{3}\left(F_{1 m}^{(k n)} \sigma_{m 2}^{(k)}-F_{m 2}^{(k n)} \sigma_{1 m}^{(k)}\right)+\frac{e_{k}}{m_{k} c^{2}}\left(F_{1 m}^{(k) r a d} \sigma_{m 2}^{(k)}-F_{m 2}^{(k) r a d} \sigma_{1 m}^{(k)}\right)$;

$\frac{d \sigma_{13}^{(k)}}{d s_{k}}=\frac{e_{k}}{m_{k} c^{2}} \sum_{n=1, n \neq k}^{3}\left(F_{1 m}^{(k n)} \sigma_{m 3}^{(k)}-F_{m 3}^{(k n)} \sigma_{1 m}^{(k)}\right)+\frac{e_{k}}{m_{k} c^{2}}\left(F_{1 m}^{(k) r a d} \sigma_{m 3}^{(k)}-F_{m 3}^{(k) r a d} \sigma_{1 m}^{(k)}\right)$

$\frac{d \sigma_{23}^{(k)}}{d s_{k}}=\frac{e_{k}}{m_{k} c^{2}} \sum_{n=1, n \neq k}^{3}\left(F_{2 m}^{(k n)} \sigma_{m 3}^{(k)}-F_{m 3}^{(k n)} \sigma_{2 m}^{(k)}\right)+\frac{e_{k}}{m_{k} c^{2}}\left(F_{2 m}^{(k) r a d} \sigma_{m 3}^{(k)}-F_{m 3}^{(k) r a d} \sigma_{2 m}^{(k)}\right)$;

$\frac{d \sigma_{14}^{(k)}}{d s_{k}}=\frac{e_{k}}{m_{k} c^{2}} \sum_{n=1, n \neq k}^{3}\left(F_{1 m}^{(k n)} \sigma_{m 4}^{(k)}-F_{m 4}^{(k n)} \sigma_{1 m}^{(k)}\right)+\frac{e_{k}}{m_{k} c^{2}}\left(F_{1 m}^{(k) r a d} \sigma_{m 4}^{(k)}-F_{m 4}^{(k) r a d} \sigma_{1 m}^{(k)}\right)$; 


$$
\begin{aligned}
\frac{d \sigma_{24}^{(k)}}{d s_{k}} & =\frac{e_{k}}{m_{k} c^{2}} \sum_{n=1, n \neq k}^{3}\left(F_{2 m}^{(k n)} \sigma_{m 4}^{(k)}-F_{m 4}^{(k n)} \sigma_{2 m}^{(k)}\right)+\frac{e_{k}}{m_{k} c^{2}}\left(F_{2 m}^{(k) r a d} \sigma_{m 4}^{(k)}-F_{m 4}^{(k) r a d} \sigma_{2 m}^{(k)}\right) ; \\
\frac{d \sigma_{34}^{(k)}}{d s_{k}} & =\frac{e_{k}}{m_{k} c^{2}} \sum_{n=1, n \neq k}^{3}\left(F_{3 m}^{(k n)} \sigma_{m 4}^{(k)}-F_{m 4}^{(k n)} \sigma_{3 m}^{(k)}\right)+\frac{e_{k}}{m_{k} c^{2}}\left(F_{3 m}^{(k) r a d} \sigma_{m 4}^{(k)}-F_{m 4}^{(k) r a d} \sigma_{3 m}^{(k)}\right) .
\end{aligned}
$$

\section{Vector Form of the Lorentz and Radiation Parts of the First Three Spin Equations}

The primary goal of the present section is to transform equations 3.1 - (3.6) and to obtain a vector form of the spin equations. Recall that $F_{i m}^{(k n)}$ are the Lorentz parts while $F_{i m}^{(k) r a d}$ - the radiation parts.

Let us consider the cross products with respect to some base $\vec{e}_{1}, \vec{e}_{2}, \vec{e}_{3}$ :

$$
\begin{gathered}
\vec{\sigma}^{(k)} \times \vec{\xi}^{(k)}=\left(\left|\begin{array}{cc}
\sigma_{2}^{(k)} & \sigma_{3}^{(k)} \\
\xi_{2}^{(k n)} & \xi_{3}^{(k n)}
\end{array}\right|,-\left|\begin{array}{cc}
\sigma_{1}^{(k)} & \sigma_{3}^{(k)} \\
\xi_{1}^{(k n)} & \xi_{3}^{(k n)}
\end{array}\right|,\left|\begin{array}{cc}
\sigma_{1}^{(k)} & \sigma_{2}^{(k)} \\
\xi_{1}^{(k n)} & \xi_{2}^{(k n)}
\end{array}\right|\right) ; \\
\vec{\lambda}^{(k)} \times \vec{\sigma}^{(k)}=\left(\left|\begin{array}{cc}
\lambda_{2}^{(k)} & \lambda_{3}^{(k)} \\
\sigma_{2}^{(k)} & \sigma_{3}^{(k)}
\end{array}\right|,-\left|\begin{array}{cc}
\lambda_{1}^{(k)} & \lambda_{3}^{(k)} \\
\sigma_{1}^{(k)} & \sigma_{3}^{(k)}
\end{array}\right|,\left|\begin{array}{cc}
\lambda_{1}^{(k)} & \lambda_{2}^{(k)} \\
\sigma_{1}^{(k)} & \sigma_{2}^{(k)}
\end{array}\right|\right) ; \\
\vec{\sigma}^{(k)} \times \vec{P}^{(k n)}=\left(\left|\begin{array}{cc}
\sigma_{2}^{(k)} & \sigma_{3}^{(k)} \\
P_{2}^{(k n)} & P_{3}^{(k n)}
\end{array}\right|,-\left|\begin{array}{cc}
\sigma_{1}^{(k)} & \sigma_{3}^{(k)} \\
P_{1}^{(k n)} & P_{3}^{(k n)}
\end{array}\right|,\left|\begin{array}{cc}
\sigma_{1}^{(k)} & \sigma_{2}^{(k)} \\
P_{1}^{(k n)} & P_{2}^{(k n)}
\end{array}\right|\right)
\end{gathered}
$$

and

$$
\begin{aligned}
\vec{P}^{(k n)} \times\left(\vec{\sigma}^{(k)} \times \vec{\xi}\right. & =\left|\begin{array}{c}
\vec{e}_{1} \\
P_{1}^{(k n)}
\end{array}=\right| \begin{array}{cc}
\vec{e}_{2} & \vec{e}_{3} \\
P_{2}^{(k n)} & P_{3}^{(k n)} \\
\sigma_{2}^{(k)} & \sigma_{3}^{(k)} \\
\xi_{2}^{(k n)} & \xi_{3}^{(k n)}
\end{array}|-| \begin{array}{cc}
\sigma_{1}^{(k)} & \sigma_{3}^{(k)} \\
\xi_{1}^{(k n)} & \xi_{3}^{(k n)}
\end{array}|| \begin{array}{cc}
\sigma_{1}^{(k)} & \sigma_{2}^{(k)} \\
\xi_{1}^{(k n)} & \xi_{2}^{(k n)}
\end{array} \mid= \\
& =\left(P_{2}{ }^{(k n)}\left|\begin{array}{cc}
\sigma_{1}^{(k)} & \sigma_{2}^{(k)} \\
\xi_{1}^{(k n)} & \xi_{2}^{(k n)}
\end{array}\right|+P_{3}{ }^{(k n)}\left|\begin{array}{cc}
\sigma_{1}^{(k)} & \sigma_{3}^{(k)} \\
\xi_{1}^{(k n)} & \xi_{3}^{(k n)}
\end{array}\right|\right) \vec{e}_{1}- \\
& -\left(P_{1}{ }^{(k n)}\left|\begin{array}{ccc}
\sigma_{1}^{(k)} & \sigma_{2}^{(k)} \\
\xi_{1}^{(k n)} & \xi_{2}^{(k n)}
\end{array}\right|-P_{3}{ }^{(k n)}\left|\begin{array}{cc}
\sigma_{2}^{(k)} & \sigma_{3}^{(k)} \\
\xi_{2}^{(k n)} & \xi_{3}^{(k n)}
\end{array}\right|\right) \vec{e}_{2}+ \\
& +\left(-P_{1}(k n)\left|\begin{array}{ccc}
\sigma_{1}^{(k)} & \sigma_{3}^{(k)} \\
\xi_{1}^{(k n)} & \xi_{3}^{(k n)}
\end{array}\right|-P_{2}^{(k n)}\left|\begin{array}{cc}
\sigma_{2}^{(k)} & \sigma_{3}^{(k)} \\
\xi_{2}^{(k n)} & \xi_{3}^{(k n)}
\end{array}\right|\right) \vec{e}_{3} .
\end{aligned}
$$

We infer

$$
\begin{aligned}
P_{2}{ }^{(k n)}\left|\begin{array}{cc}
\sigma_{1}^{(k)} & \sigma_{2}^{(k)} \\
\xi_{1}^{(k n)} & \xi_{2}^{(k n)}
\end{array}\right|+P_{3}{ }^{(k n)}\left|\begin{array}{cc}
\sigma_{1}^{(k)} & \sigma_{3}^{(k)} \\
\xi_{1}^{(k n)} & \xi_{3}^{(k n)}
\end{array}\right|=\left[\vec{P}^{(k n)} \times\left(\vec{\sigma}^{(k)} \times \vec{\xi}^{(k)}\right)\right]_{1} ; \\
-P_{1}{ }^{(k n)}\left|\begin{array}{cc}
\sigma_{1}^{(k)} & \sigma_{2}^{(k)} \\
\xi_{1}^{(k n)} & \xi_{2}^{(k n)}
\end{array}\right|+P_{3}^{(k n)}\left|\begin{array}{cc}
\sigma_{2}^{(k)} & \sigma_{3}^{(k)} \\
\xi_{2}^{(k n)} & \xi_{3}^{(k n)}
\end{array}\right|=\left[\vec{P}^{(k n)} \times\left(\vec{\sigma}^{(k)} \times \vec{\xi}^{(k)}\right)\right]_{2} ; \\
-P_{1}^{(k n)}\left|\begin{array}{cc}
\sigma_{1}^{(k)} & \sigma_{3}^{(k)} \\
\xi_{1}^{(k n)} & \xi_{3}^{(k n)}
\end{array}\right|-P_{2}^{(k n)}\left|\begin{array}{cc}
\sigma_{2}^{(k)} & \sigma_{3}^{(k)} \\
\xi_{2}^{(k n)} & \xi_{3}^{(k n)}
\end{array}\right|=\left[\vec{P}^{(k n)} \times\left(\vec{\sigma}^{(k)} \times \vec{\xi}^{(k)}\right)\right]_{3}
\end{aligned}
$$

where the right subscripts mean the number of the coordinate. In the same way

$$
\vec{P}^{(k n)} \times\left(\vec{\lambda}^{(k)} \times \vec{\sigma}^{(k)}\right)=\left|\begin{array}{ccc}
\vec{e}_{1} & \vec{e}_{2} & \vec{e}_{3} \\
P_{1}^{(k n)} & P_{2}^{(k n)} & P_{3}^{(k n)} \\
\mid \lambda_{2}^{(k)} & \lambda_{3}^{(k)} \\
\sigma_{2}^{(k)} & \sigma_{3}^{(k)}
\end{array}\right|-\left|\begin{array}{cc}
\lambda_{1}^{(k)} & \lambda_{3}^{(k)} \\
\sigma_{1}^{(k)} & \sigma_{3}^{(k)}
\end{array}\right|\left|\begin{array}{cc}
\lambda_{1}^{(k)} & \lambda_{2}^{(k)} \\
\sigma_{1}^{(k)} & \sigma_{2}^{(k)}
\end{array}\right| \mid=
$$




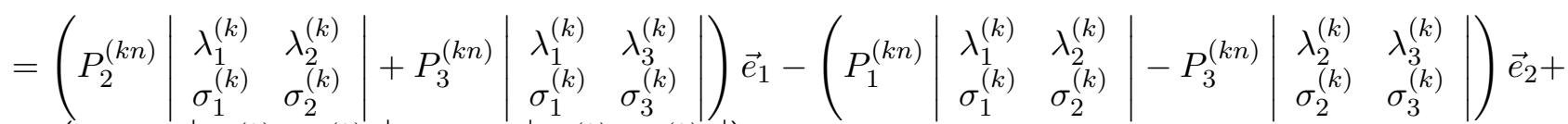

$$
\begin{aligned}
& +\left(-P_{1}^{(k n)}\left|\begin{array}{cc}
\lambda_{1}^{(k)} & \lambda_{3}^{(k)} \\
\sigma_{1}^{(k)} & \sigma_{3}^{(k)}
\end{array}\right|-P_{2}^{(k n)}\left|\begin{array}{cc}
\lambda_{2}^{(k)} & \lambda_{3}^{(k)} \\
\sigma_{2}^{(k)} & \sigma_{3}^{(k)}
\end{array}\right|\right) \vec{e}_{3}
\end{aligned}
$$

that means

$$
\begin{gathered}
P_{2}^{(k n)}\left|\begin{array}{cc}
\lambda_{1}^{(k)} & \lambda_{2}^{(k)} \\
\sigma_{1}^{(k)} & \sigma_{2}^{(k)}
\end{array}\right|+P_{3}^{(k n)}\left|\begin{array}{cc}
\lambda_{1}^{(k)} & \lambda_{3}^{(k)} \\
\sigma_{1}^{(k)} & \sigma_{3}^{(k)}
\end{array}\right|=\left[\vec{P}^{(k n)} \times\left(\vec{\lambda}^{(k)} \times \vec{\sigma}^{(k)}\right)\right]_{1} ; \\
-P_{1}^{(k n)}\left|\begin{array}{cc}
\lambda_{1}^{(k)} & \lambda_{2}^{(k)} \\
\sigma_{1}^{(k)} & \sigma_{2}^{(k)}
\end{array}\right|+P_{3}^{(k n)}\left|\begin{array}{cc}
\lambda_{2}^{(k)} & \lambda_{3}^{(k)} \\
\sigma_{2}^{(k)} & \sigma_{3}^{(k)}
\end{array}\right|=\left[\vec{P}^{(k n)} \times\left(\vec{\lambda}^{(k)} \times \vec{\sigma}^{(k)}\right)\right]_{2} ; \\
-P_{1}^{(k n)}\left|\begin{array}{cc}
\lambda_{1}^{(k)} & \lambda_{3}^{(k)} \\
\sigma_{1}^{(k)} & \sigma_{3}^{(k)}
\end{array}\right|-P_{2}^{(k n)}\left|\begin{array}{cc}
\lambda_{2}^{(k)} & \lambda_{3}^{(k)} \\
\sigma_{2}^{(k)} & \sigma_{3}^{(k)}
\end{array}\right|=\left[\vec{P}^{(k n)} \times\left(\vec{\lambda}^{(k)} \times \vec{\sigma}^{(k)}\right)\right]_{3} ;
\end{gathered}
$$

For the next term

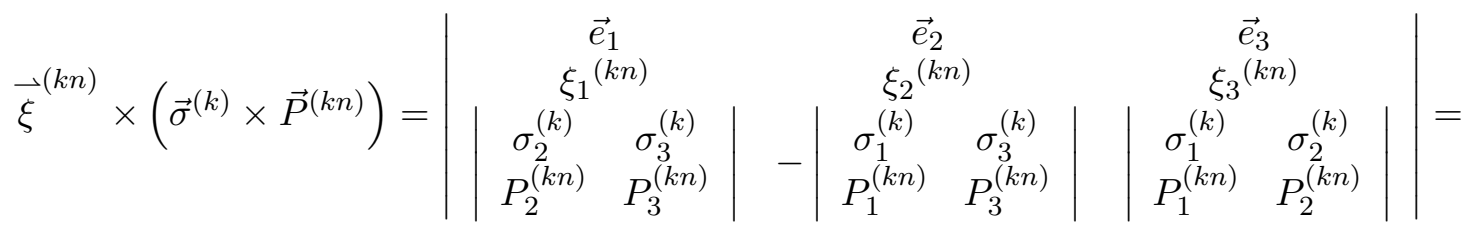

$$
\begin{aligned}
& =\left(\xi_{2}(k n)\left|\begin{array}{cc}
\sigma_{1}^{(k)} & \sigma_{2}^{(k)} \\
P_{1}^{(k n)} & P_{2}^{(k n)}
\end{array}\right|+\xi_{3}(k n)\left|\begin{array}{cc}
\sigma_{1}^{(k)} & \sigma_{3}^{(k)} \\
P_{1}^{(k n)} & P_{3}^{(k n)}
\end{array}\right|\right) \vec{e}_{1}- \\
& -\left(\xi_{1}{ }^{(k n)}\left|\begin{array}{cc}
\sigma_{1}^{(k)} & \sigma_{2}^{(k)} \\
P_{1}^{(k n)} & P_{2}^{(k n)}
\end{array}\right|-\xi_{3}{ }^{(k n)}\left|\begin{array}{cc}
\sigma_{2}^{(k)} & \sigma_{3}^{(k)} \\
P_{2}^{(k n)} & P_{3}^{(k n)}
\end{array}\right|\right) \vec{e}_{2}+ \\
& +\left(-\xi_{1}(k n)\left|\begin{array}{cc}
\sigma_{1}^{(k)} & \sigma_{3}^{(k)} \\
P_{1}^{(k n)} & P_{3}^{(k n)}
\end{array}\right|-\xi_{2}(k n)\left|\begin{array}{cc}
\sigma_{2}^{(k)} & \sigma_{3}^{(k)} \\
P_{2}^{(k n)} & P_{3}^{(k n)}
\end{array}\right|\right) \vec{e}_{3} .
\end{aligned}
$$

Therefore

$$
\begin{aligned}
& \xi_{2}{ }^{(k n)}\left|\begin{array}{cc}
\sigma_{1}^{(k)} & \sigma_{2}^{(k)} \\
P_{1}^{(k n)} & P_{2}^{(k n)}
\end{array}\right|+\xi_{3}{ }^{(k n)}\left|\begin{array}{cc}
\sigma_{1}^{(k)} & \sigma_{3}^{(k)} \\
P_{1}^{(k n)} & P_{3}^{(k n)}
\end{array}\right|=\left[\vec{\xi}^{(k n)} \times\left(\vec{\sigma}^{(k)} \times \vec{P}^{(k)}\right)\right]_{1} ;
\end{aligned}
$$

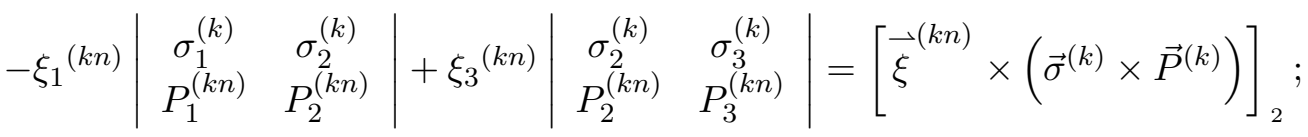

$$
\begin{aligned}
& -\xi_{1}^{(k n)}\left|\begin{array}{cc}
\sigma_{1}^{(k)} & \sigma_{3}^{(k)} \\
P_{1}^{(k n)} & P_{3}^{(k n)}
\end{array}\right|-\xi_{2}^{(k n)}\left|\begin{array}{cc}
\sigma_{2}^{(k)} & \sigma_{3}^{(k)} \\
P_{2}^{(k n)} & P_{3}^{(k n)}
\end{array}\right|=\left[\vec{\xi}^{(k n)} \times\left(\vec{\sigma}^{(k)} \times \vec{P}^{(k)}\right)\right]_{3}
\end{aligned}
$$

and finally

$$
\begin{aligned}
& \vec{\xi}^{(k n)} \times\left(\vec{\lambda}^{(k)} \times \vec{\sigma}^{(k)}\right)=\left|\begin{array}{ccc}
\vec{e}_{1} & \vec{e}_{2} & \vec{e}_{3} \\
\xi_{1}(k n) & \xi_{2}^{(k n)} & \xi_{3}(k n) \\
\lambda_{2}^{(k)} & \lambda_{3}^{(k)} \\
\sigma_{2}^{(k)} & \sigma_{3}^{(k)}
\end{array}\right|-\left|\begin{array}{cc}
\lambda_{1}^{(k)} & \lambda_{3}^{(k)} \\
\sigma_{1}^{(k)} & \sigma_{3}^{(k)}
\end{array}\right|\left|\begin{array}{cc}
\lambda_{1}^{(k)} & \lambda_{2}^{(k)} \\
\sigma_{1}^{(k)} & \sigma_{2}^{(k)}
\end{array}\right| \mid= \\
& =\left(\xi_{2}(k n)\left|\begin{array}{cc}
\lambda_{1}^{(k)} & \lambda_{2}^{(k)} \\
\sigma_{1}^{(k)} & \sigma_{2}^{(k)}
\end{array}\right|+\xi_{3}{ }^{(k n)}\left|\begin{array}{cc}
\lambda_{1}^{(k)} & \lambda_{3}^{(k)} \\
\sigma_{1}^{(k)} & \sigma_{3}^{(k)}
\end{array}\right|\right) \vec{e}_{1}-\left(\xi_{1}{ }^{(k n)}\left|\begin{array}{cc}
\lambda_{1}^{(k)} & \lambda_{2}^{(k)} \\
\sigma_{1}^{(k)} & \sigma_{2}^{(k)}
\end{array}\right|-\xi_{3}(k n)\left|\begin{array}{cc}
\lambda_{2}^{(k)} & \lambda_{3}^{(k)} \\
\sigma_{2}^{(k)} & \sigma_{3}^{(k)}
\end{array}\right|\right) \vec{e}_{2}+ \\
& +\left(-\xi_{1}(k n)\left|\begin{array}{cc}
\lambda_{1}^{(k)} & \lambda_{3}^{(k)} \\
\sigma_{1}^{(k)} & \sigma_{3}^{(k)}
\end{array}\right|-\xi_{2}(k n)\left|\begin{array}{cc}
\lambda_{2}^{(k)} & \lambda_{3}^{(k)} \\
\sigma_{2}^{(k)} & \sigma_{3}^{(k)}
\end{array}\right|\right) \vec{e}_{3}
\end{aligned}
$$


that is,

$$
\begin{aligned}
& \xi_{2}^{(k n)}\left|\begin{array}{cc}
\lambda_{1}^{(k)} & \lambda_{2}^{(k)} \\
\sigma_{1}^{(k)} & \sigma_{2}^{(k)}
\end{array}\right|+\xi_{3}^{(k n)}\left|\begin{array}{cc}
\lambda_{1}^{(k)} & \lambda_{3}^{(k)} \\
\sigma_{1}^{(k)} & \sigma_{3}^{(k)}
\end{array}\right|=\left[\vec{\xi}^{(k n)} \times\left(\vec{\lambda}^{(k)} \times \vec{\sigma}^{(k)}\right)\right]_{1} ; \\
& -\xi_{1}^{(k n)}\left|\begin{array}{cc}
\lambda_{1}^{(k)} & \lambda_{2}^{(k)} \\
\sigma_{1}^{(k)} & \sigma_{2}^{(k)}
\end{array}\right|+\xi_{3}^{(k n)}\left|\begin{array}{cc}
\lambda_{2}^{(k)} & \lambda_{3}^{(k)} \\
\sigma_{2}^{(k)} & \sigma_{3}^{(k)}
\end{array}\right|=\left[\vec{\xi}^{(k n)} \times\left(\vec{\lambda}^{(k)} \times \vec{\sigma}^{(k)}\right)\right]_{2} ; \\
& -\xi_{1}^{(k n)}\left|\begin{array}{cc}
\lambda_{1}^{(k)} & \lambda_{3}^{(k)} \\
\sigma_{1}^{(k)} & \sigma_{3}^{(k)}
\end{array}\right|-\xi_{2}^{(k n)}\left|\begin{array}{cc}
\lambda_{2}^{(k)} & \lambda_{3}^{(k)} \\
\sigma_{2}^{(k)} & \sigma_{3}^{(k)}
\end{array}\right|=\left[\vec{\xi}^{(k n)} \times\left(\vec{\lambda}^{(k)} \times \vec{\sigma}^{(k)}\right)\right]_{3} .
\end{aligned}
$$

Transformation of the Lorentz Part of Equation 3.1

First, we transform the Lorentz part, because the radiation parts can be transformed in the same way. So instead of

$$
\frac{d \sigma_{12}^{(k)}}{d s_{k}}=\frac{e_{k}}{m_{k} c^{2}} \sum_{n=1, n \neq k}^{3}\left(F_{1 m}^{(k n)} \sigma_{m 2}^{(k)}-\sigma_{1 m}^{(k)} F_{m 2}^{(k n)}\right)+\frac{e_{k}}{m_{k} c^{2}}\left(F_{1 m}^{(k) r a d} \sigma_{m 2}^{(k)}-\sigma_{1 m}^{(k)} F_{m 2}^{(k) r a d}\right)
$$

we consider only

$$
\begin{gathered}
\frac{d \sigma_{12}^{(k)}}{d s_{k}}=\sum_{n=1, n \neq k}^{3} \frac{e_{k} e_{n}}{m_{k} c^{2}}\left[\sigma_{m 2}^{(k)}\left(P_{1}^{(k n)} \xi_{m}^{(k n)}-P_{m}^{(k n)} \xi_{1}^{(k n)}\right)-\sigma_{1 m}^{(k)}\left(P_{m}^{(k n)} \xi_{2}^{(k n)}-P_{2}^{(k n)} \xi_{m}^{(k n)}\right)\right]= \\
=\sum_{n=1, n \neq k}^{3} \frac{e_{k} e_{n}}{m_{k} c^{2}}\left(P_{1}^{(k n)} \sigma_{m 2}^{(k)} \xi_{m}^{(k n)}-\xi_{1}^{(k n)} \sigma_{m 2}^{(k)} P_{m}^{(k n)}-\xi_{2}^{(k n)} \sigma_{1 m}^{(k)} P_{m}^{(k n)}+P_{2}^{(k n)} \sigma_{1 m}^{(k)} \xi_{m}^{(k n)}\right) .
\end{gathered}
$$

But

$$
\begin{aligned}
& P_{1}^{(k n)} \sigma_{m 2}^{(k)} \xi_{m}^{(k n)}=P_{1}^{(k n)}\left(\sigma_{12}^{(k)} \xi_{1}^{(k n)}+\sigma_{32}^{(k)} \xi_{3}^{(k n)}+\sigma_{42}^{(k)} \xi_{4}^{(k n)}\right)=P_{1}^{(k n)}\left(\sigma_{3}^{(k)} \xi_{1}^{(k n)}-\sigma_{1}^{(k)} \xi_{3}^{(k n)}+\sigma_{42}^{(k)} \xi_{4}^{(k n)}\right)= \\
& =-P_{1}^{(k n)}\left|\begin{array}{cc}
\sigma_{1}^{(k)} & \sigma_{3}^{(k)} \\
\xi_{1}^{(k n)} & \xi_{3}^{(k n)}
\end{array}\right|-P_{1}^{(k n)} \tau_{k n}\left|\begin{array}{cc}
\lambda_{1}^{(k)} & \lambda_{3}^{(k)} \\
\sigma_{1}^{(k)} & \sigma_{3}^{(k)}
\end{array}\right| ; \\
& -\xi_{1}^{(k n)} \sigma_{m 2}^{(k)} P_{m}^{(k n)}=-\xi_{1}^{(k n)}\left(\sigma_{12}^{(k)} P_{1}^{(k n)}+\sigma_{32}^{(k)} P_{3}^{(k n)}+\sigma_{42}^{(k)} P_{4}^{(k n)}\right) \\
& =-\xi_{1}^{(k n)}\left(\sigma_{3}^{(k)} P_{1}^{(k n)}-\sigma_{1}^{(k)} P_{3}^{(k n)}+\sigma_{42}^{(k)} P_{4}^{(k n)}\right)=\xi_{1}^{(k n)}\left|\begin{array}{cc}
\sigma_{1}^{(k)} & \sigma_{3}^{(k)} \\
P_{1}^{(k n)} & P_{3}^{(k n)}
\end{array}\right|-\xi_{1}^{(k n)} L_{k n}\left|\begin{array}{cc}
\lambda_{1}^{(k)} & \lambda_{3}^{(k)} \\
\sigma_{1}^{(k)} & \sigma_{3}^{(k)}
\end{array}\right| ; \\
& -\xi_{2}^{(k n)} \sigma_{1 m}^{(k)} P_{m}^{(k n)}=-\xi_{2}^{(k n)}\left(\sigma_{12}^{(k)} P_{2}^{(k n)}+\sigma_{13}^{(k)} P_{3}^{(k n)}+\sigma_{14}^{(k)} P_{4}^{(k n)}\right)= \\
& =-\xi_{2}^{(k n)}\left(\sigma_{3}^{(k)} P_{2}^{(k n)}-\sigma_{2}^{(k)} P_{3}^{(k n)}+\sigma_{14}^{(k)} P_{4}^{(k n)}\right)=\xi_{2}^{(k n)}\left|\begin{array}{cc}
\sigma_{2}^{(k)} & \sigma_{3}^{(k)} \\
P_{2}^{(k n)} & P_{3}^{(k n)}
\end{array}\right|-\xi_{2}^{(k n)} L_{k n}\left|\begin{array}{cc}
\lambda_{2}^{(k)} & \lambda_{3}^{(k)} \\
\sigma_{2}^{(k)} & \sigma_{3}^{(k)}
\end{array}\right| ; \\
& P_{2}^{(k n)} \sigma_{1 m}^{(k)} \xi_{m}^{(k n)}=P_{2}^{(k n)}\left(\sigma_{12}^{(k)} \xi_{2}^{(k n)}+\sigma_{13}^{(k)} \xi_{3}^{(k n)}+\sigma_{14}^{(k)} \xi_{4}^{(k n)}\right)=P_{2}^{(k n)}\left(\sigma_{3}^{(k)} \xi_{2}^{(k n)}-\sigma_{2}^{(k)} \xi_{3}^{(k n)}+\sigma_{14}^{(k)} \xi_{4}^{(k n)}\right)= \\
& =-P_{2}^{(k n)}\left|\begin{array}{cc}
\sigma_{2}^{(k)} & \sigma_{3}^{(k)} \\
\xi_{2}^{(k n)} & \xi_{3}^{(k n)}
\end{array}\right|-\tau_{k n} P_{2}^{(k n)}\left|\begin{array}{cc}
\lambda_{2}^{(k)} & \lambda_{3}^{(k)} \\
\sigma_{2}^{(k)} & \sigma_{3}^{(k)}
\end{array}\right|
\end{aligned}
$$

and then in view of $\frac{d \sigma_{12}^{(k)}}{d s_{k}}=\frac{d \sigma_{3}^{(k)}}{d s_{k}}$ we obtain 


$$
\begin{aligned}
\frac{d \sigma_{3}^{(k)}}{d s_{k}} & =\sum_{n=1, n \neq k}^{3} \frac{e_{k} e_{n}}{m_{k} c^{2}}\left(-P_{1}^{(k n)}\left|\begin{array}{cc}
\sigma_{1}^{(k)} & \sigma_{3}^{(k)} \\
\xi_{1}^{(k n)} & \xi_{3}^{(k n)}
\end{array}\right|-\tau_{k n} P_{1}^{(k n)}\left|\begin{array}{cc}
\lambda_{1}^{(k)} & \lambda_{3}^{(k)} \\
\sigma_{1}^{(k)} & \sigma_{3}^{(k)}
\end{array}\right|+\xi_{1}^{(k n)}\left|\begin{array}{cc}
\sigma_{1}^{(k)} & \sigma_{3}^{(k)} \\
P_{1}^{(k n)} & P_{3}^{(k n)}
\end{array}\right|-\right. \\
& -L_{k n} \xi_{1}^{(k n)}\left|\begin{array}{cc}
\lambda_{1}^{(k)} & \lambda_{3}^{(k)} \\
\sigma_{1}^{(k)} & \sigma_{3}^{(k)}
\end{array}\right|+\xi_{2}^{(k n)}\left|\begin{array}{cc}
\sigma_{2}^{(k)} & \sigma_{3}^{(k)} \\
P_{2}^{(k n)} & P_{3}^{(k n)}
\end{array}\right|-L_{k n} \xi_{2}^{(k n)}\left|\begin{array}{cc}
\lambda_{2}^{(k)} & \lambda_{3}^{(k)} \\
\sigma_{2}^{(k)} & \sigma_{3}^{(k)}
\end{array}\right|- \\
& \left.-P_{2}^{(k n)}\left|\begin{array}{cc}
\sigma_{2}^{(k)} & \sigma_{3}^{(k)} \\
\xi_{2}^{(k n)} & \xi_{3}^{(k n)}
\end{array}\right|-\tau_{k n} P_{2}^{(k n)}\left|\begin{array}{cc}
\lambda_{2}^{(k)} & \lambda_{3}^{(k)} \\
\sigma_{2}^{(k)} & \sigma_{3}^{(k)}
\end{array}\right|\right)
\end{aligned}
$$

or

$$
\begin{aligned}
& \frac{d \sigma_{3}^{(k)}}{d s_{k}}=\sum_{n=1, n \neq k}^{3} \frac{e_{k} e_{n}}{m_{k} c^{2}}\left(-P_{1}^{(k n)}\left|\begin{array}{cc}
\sigma_{1}^{(k)} & \sigma_{3}^{(k)} \\
\xi_{1}^{(k n)} & \xi_{3}^{(k n)}
\end{array}\right|-P_{2}^{(k n)}\left|\begin{array}{cc}
\sigma_{2}^{(k)} & \sigma_{3}^{(k)} \\
\xi_{2}^{(k n)} & \xi_{3}^{(k n)}
\end{array}\right|-\tau_{k n} P_{1}^{(k n)}\left|\begin{array}{cc}
\lambda_{1}^{(k)} & \lambda_{3}^{(k)} \\
\sigma_{1}^{(k)} & \sigma_{3}^{(k)}
\end{array}\right|-\right. \\
& -\tau_{k n} P_{2}^{(k n)}\left|\begin{array}{cc}
\lambda_{2}^{(k)} & \lambda_{3}^{(k)} \\
\sigma_{2}^{(k)} & \sigma_{3}^{(k)}
\end{array}\right|+\xi_{1}^{(k n)}\left|\begin{array}{cc}
\sigma_{1}^{(k)} & \sigma_{3}^{(k)} \\
P_{1}^{(k n)} & P_{3}^{(k n)}
\end{array}\right|+\xi_{2}^{(k n)}\left|\begin{array}{cc}
\sigma_{2}^{(k)} & \sigma_{3}^{(k)} \\
P_{2}^{(k n)} & P_{3}^{(k n)}
\end{array}\right|-L_{k n} \xi_{1}^{(k n)}\left|\begin{array}{cc}
\lambda_{1}^{(k)} & \lambda_{3}^{(k)} \\
\sigma_{1}^{(k)} & \sigma_{3}^{(k)}
\end{array}\right|- \\
& \left.-L_{k n} \xi_{2}^{(k n)}\left|\begin{array}{cc}
\lambda_{2}^{(k)} & \lambda_{3}^{(k)} \\
\sigma_{2}^{(k)} & \sigma_{3}^{(k)}
\end{array}\right|\right)
\end{aligned}
$$

and consequently

$$
\begin{aligned}
& \frac{d \sigma_{3}^{(k)}}{d s_{k}}= \\
& \sum_{n=1, n \neq k}^{3} \frac{e_{k} e_{n}}{m_{k} c^{2}}\left\{\left[\vec{P}^{(k n)} \times\left(\vec{\sigma}^{(k)} \times \vec{\xi}^{(k n)}\right)\right]_{3}+\tau_{k n}\left[\vec{P}^{(k n)} \times\left(\vec{\lambda}^{(k)} \times \vec{\sigma}^{(k)}\right)\right]_{3}-\left[\vec{\xi}^{(k n)} \times\left(\vec{\sigma}^{(k)} \times \vec{P}^{(k n)}\right)\right]_{3}+\right. \\
& \left.\quad+L_{k n}\left[\vec{\xi}^{(k n)} \times\left(\vec{\lambda}^{(k)} \times \vec{\sigma}^{(k)}\right)\right]_{3}\right\} .
\end{aligned}
$$

Transformation of the Lorentz Part of Equation 3.2

Here

$$
\begin{aligned}
& \frac{d \sigma_{13}^{(k)}}{d s_{k}}=\frac{e_{k}}{m_{k} c^{2}} \sum_{n=1, n \neq k}^{3}\left(F_{1 m}^{(k n)} \sigma_{m 3}^{(k)}-\sigma_{1 m}^{(k)} F_{m 3}^{(k n)}\right)+\frac{e_{k}}{m_{k} c^{2}}\left(F_{1 m}^{(k) r a d} \sigma_{m 3}^{(k)}-\sigma_{1 m}^{(k)} F_{m 3}^{(k) r a d}\right) \\
& \frac{d \sigma_{13}^{(k)}}{d s_{k}}=\frac{e_{k}}{m_{k} c^{2}} \sum_{n=1, n \neq k}^{3}\left(F_{1 m}^{(k n)} \sigma_{m 3}^{(k)}-\sigma_{1 m}^{(k)} F_{m 3}^{(k n)}\right) \\
& =\sum_{n=1, n \neq k}^{3} \frac{e_{k} e_{n}}{m_{k} c^{2}}\left[\sigma_{m 3}^{(k)}\left(P_{1}^{(k n)} \xi_{m}^{(k n)}-P_{m}^{(k n)} \xi_{1}^{(k n)}\right)-\sigma_{1 m}^{(k)}\left(P_{m}^{(k n)} \xi_{3}^{(k n)}-P_{3}^{(k n)} \xi_{m}^{(k n)}\right)\right] \\
& =\sum_{n=1, n \neq k}^{3} \frac{e_{k} e_{n}}{m_{k} c^{2}}\left(P_{1}^{(k n)} \sigma_{m 3}^{(k)} \xi_{m}^{(k n)}-\sigma_{m 3}^{(k)} P_{m}^{(k n)} \xi_{1}^{(k n)}-\sigma_{1 m}^{(k)} P_{m}^{(k n)} \xi_{3}^{(k n)}+P_{3}^{(k n)} \sigma_{1 m}^{(k)} \xi_{m}^{(k n)}\right) ; \\
& P_{1}^{(k n)} \sigma_{m 3}^{(k)} \xi_{m}^{(k n)}=P_{1}^{(k n)}\left(\sigma_{13}^{(k)} \xi_{1}^{(k n)}+\sigma_{23}^{(k)} \xi_{2}^{(k n)}+\sigma_{43}^{(k)} \xi_{4}^{(k n)}\right)=P_{1}^{(k n)}\left(-\sigma_{2}^{(k)} \xi_{1}^{(k n)}+\sigma_{1}^{(k)} \xi_{2}^{(k n)}+\sigma_{43}^{(k)} \xi_{4}^{(k n)}\right) \\
& =P_{1}^{(k n)}\left|\begin{array}{cc}
\sigma_{1}^{(k)} & \sigma_{2}^{(k)} \\
\xi_{1}^{(k n)} & \xi_{2}^{(k n)}
\end{array}\right|+\tau_{k n} P_{1}^{(k n)}\left|\begin{array}{cc}
\lambda_{1}^{(k)} & \lambda_{2}^{(k)} \\
\sigma_{1}^{(k)} & \sigma_{2}^{(k)}
\end{array}\right| ; \\
& -\xi_{1}^{(k n)} \sigma_{m 3}^{(k)} P_{m}^{(k n)}=-\xi_{1}^{(k n)}\left(\sigma_{13}^{(k)} P_{1}^{(k n)}+\sigma_{23}^{(k)} P_{2}^{(k n)}+\sigma_{43}^{(k)} P_{4}^{(k n)}\right)=-\xi_{1}^{(k n)}\left(-\sigma_{2}^{(k)} P_{1}^{(k n)}+\sigma_{1}^{(k)} P_{2}^{(k n)}+\sigma_{43}^{(k)} P_{4}^{(k n)}\right) \\
& =-\xi_{1}^{(k n)}\left|\begin{array}{cc}
\sigma_{1}^{(k)} & \sigma_{2}^{(k)} \\
P_{1}^{(k n)} & P_{2}^{(k n)}
\end{array}\right|+L_{k n} \xi_{1}^{(k n)}\left|\begin{array}{cc}
\lambda_{1}^{(k)} & \lambda_{2}^{(k)} \\
\sigma_{1}^{(k)} & \sigma_{2}^{(k)}
\end{array}\right| ; \\
& -\xi_{3}^{(k n)} \sigma_{1 m}^{(k)} P_{m}^{(k n)}=-\xi_{3}^{(k n)}\left(\sigma_{12}^{(k)} P_{2}^{(k n)}+\sigma_{13}^{(k)} P_{3}^{(k n)}+\sigma_{14}^{(k)} P_{4}^{(k n)}\right)=-\xi_{3}^{(k n)}\left(\sigma_{3}^{(k)} P_{2}^{(k n)}-\sigma_{2}^{(k)} P_{3}^{(k n)}+\sigma_{14}^{(k)} P_{4}^{(k n)}\right)
\end{aligned}
$$




$$
\begin{aligned}
& =\xi_{3}^{(k n)}\left|\begin{array}{cc}
\sigma_{2}^{(k)} & \sigma_{3}^{(k)} \\
P_{2}^{(k n)} & P_{3}^{(k n)}
\end{array}\right|-L_{k n} \xi_{3}^{(k n)}\left|\begin{array}{cc}
\lambda_{2}^{(k)} & \lambda_{3}^{(k)} \\
\sigma_{2}^{(k)} & \sigma_{3}^{(k)}
\end{array}\right| ; \\
& P_{3}^{(k n)} \sigma_{1 m}^{(k)} \xi_{m}^{(k n)}=P_{3}^{(k n)}\left(\sigma_{12}^{(k)} \xi_{2}^{(k n)}+\sigma_{13}^{(k)} \xi_{3}^{(k n)}+\sigma_{14}^{(k)} \xi_{4}^{(k n)}\right)=P_{3}^{(k n)}\left(\sigma_{3}^{(k)} \xi_{2}^{(k n)}-\sigma_{2}^{(k)} \xi_{3}^{(k n)}+\sigma_{14}^{(k)} \xi_{4}^{(k n)}\right)= \\
& =-P_{3}^{(k n)}\left|\begin{array}{cc}
\sigma_{2}^{(k)} & \sigma_{3}^{(k)} \\
\xi_{2}^{(k n)} & \xi_{3}^{(k n)}
\end{array}\right|-\tau_{k n} P_{3}^{(k n)}\left|\begin{array}{cc}
\lambda_{2}^{(k)} & \lambda_{3}^{(k)} \\
\sigma_{2}^{(k)} & \sigma_{3}^{(k)}
\end{array}\right| .
\end{aligned}
$$

Then

$$
\begin{gathered}
\sum_{n=1, n \neq k}^{3}\left(F_{1 m}^{(k n)} \sigma_{m 3}^{(k)}-\sigma_{1 m}^{(k)} F_{m 3}^{(k n)}\right)= \\
=\sum_{n=1, n \neq k}^{3} e_{n}\left(P_{1}^{(k n)}\left|\begin{array}{cc}
\sigma_{1}^{(k)} & \sigma_{2}^{(k)} \\
\xi_{1}^{(k n)} & \xi_{2}^{(k n)}
\end{array}\right|+\tau_{k n} P_{1}^{(k n)}\left|\begin{array}{cc}
\lambda_{1}^{(k)} & \lambda_{2}^{(k)} \\
\sigma_{1}^{(k)} & \sigma_{2}^{(k)}
\end{array}\right|-\xi_{1}^{(k n)}\left|\begin{array}{cc}
\sigma_{1}^{(k)} & \sigma_{2}^{(k)} \\
P_{1}^{(k n)} & P_{2}^{(k n)}
\end{array}\right|+\right. \\
+L_{k n} \xi_{1}^{(k n)}\left|\begin{array}{cc}
\lambda_{1}^{(k)} & \lambda_{2}^{(k)} \\
\sigma_{1}^{(k)} & \sigma_{2}^{(k)} \\
\lambda_{2}^{(k)} & \lambda_{3}^{(k)} \\
\sigma_{2}^{(k)} & \sigma_{3}^{(k)}
\end{array}\right|++\xi_{3}^{(k n)}\left|\begin{array}{cc}
\sigma_{2}^{(k)} & \sigma_{3}^{(k)} \\
P_{2}^{(k n)} & P_{3}^{(k n)}
\end{array}\right|-L_{k n} \xi_{3}^{(k n)}\left|\begin{array}{cc}
\lambda_{2}^{(k)} & \lambda_{3}^{(k)} \\
\sigma_{2}^{(k)} & \sigma_{3}^{(k)}
\end{array}\right|-P_{3}^{(k n)}\left|\begin{array}{cc}
\sigma_{2}^{(k)} & \sigma_{3}^{(k)} \\
\xi_{2}^{(k n)} & \xi_{3}^{(k n)}
\end{array}\right|- \\
-\tau_{k n} P_{3}^{(k n)}
\end{gathered}
$$

In view of $\frac{d \sigma_{13}^{(k)}}{d s_{k}}=-\frac{d \sigma_{2}^{(k)}}{d s_{k}}$ we have

$$
\begin{aligned}
& -\frac{d \sigma_{2}^{(k)}}{d s_{k}}=\sum_{n=1, n \neq k}^{3} \frac{e_{k} e_{n}}{m_{k} c^{2}}\left(P_{1}^{(k n)}\left|\begin{array}{cc}
\sigma_{1}^{(k)} & \sigma_{2}^{(k)} \\
\xi_{1}^{(k n)} & \xi_{2}^{(k n)}
\end{array}\right|-P_{3}^{(k n)}\left|\begin{array}{cc}
\sigma_{2}^{(k)} & \sigma_{3}^{(k)} \\
\xi_{2}^{(k n)} & \xi_{3}^{(k n)}
\end{array}\right|+\tau_{k n} P_{1}^{(k n)}\left|\begin{array}{cc}
\lambda_{1}^{(k)} & \lambda_{2}^{(k)} \\
\sigma_{1}^{(k)} & \sigma_{2}^{(k)}
\end{array}\right|-\right. \\
& -\tau_{k n} P_{3}^{(k n)}\left|\begin{array}{cc}
\lambda_{2}^{(k)} & \lambda_{3}^{(k)} \\
\sigma_{2}^{(k)} & \sigma_{3}^{(k)}
\end{array}\right|-\xi_{1}^{(k n)}\left|\begin{array}{cc}
\sigma_{1}^{(k)} & \sigma_{2}^{(k)} \\
P_{1}^{(k n)} & P_{2}^{(k n)}
\end{array}\right|+\xi_{3}^{(k n)}\left|\begin{array}{cc}
\sigma_{2}^{(k)} & \sigma_{3}^{(k)} \\
P_{2}^{(k n)} & P_{3}^{(k n)}
\end{array}\right|+ \\
& \left.+L_{k n} \xi_{1}^{(k n)}\left|\begin{array}{cc}
\lambda_{1}^{(k)} & \lambda_{2}^{(k)} \\
\sigma_{1}^{(k)} & \sigma_{2}^{(k)}
\end{array}\right|-L_{k n} \xi_{3}^{(k n)}\left|\begin{array}{cc}
\lambda_{2}^{(k)} & \lambda_{3}^{(k)} \\
\sigma_{2}^{(k)} & \sigma_{3}^{(k)}
\end{array}\right|\right) \text {. }
\end{aligned}
$$

Therefore

$$
\begin{aligned}
& P_{1}^{(k n)}\left|\begin{array}{cc}
\sigma_{1}^{(k)} & \sigma_{2}^{(k)} \\
\xi_{1}^{(k n)} & \xi_{2}^{(k n)}
\end{array}\right|-P_{3}^{(k n)}\left|\begin{array}{cc}
\sigma_{2}^{(k)} & \sigma_{3}^{(k)} \\
\xi_{2}^{(k n)} & \xi_{3}^{(k n)}
\end{array}\right|=-\left[\vec{P}^{(k n)} \times\left(\vec{\sigma}^{(k)} \times \vec{\xi}^{(k)}\right)\right]_{2} ; \\
& \tau_{k n} P_{1}^{(k n)}\left|\begin{array}{cc}
\lambda_{1}^{(k)} & \lambda_{2}^{(k)} \\
\sigma_{1}^{(k)} & \sigma_{2}^{(k)}
\end{array}\right|-\tau_{k n} P_{3}^{(k n)}\left|\begin{array}{cc}
\lambda_{1}^{(k)} & \lambda_{2}^{(k)} \\
\sigma_{1}^{(k)} & \sigma_{2}^{(k)}
\end{array}\right|=-\tau_{k n}\left[\vec{P}^{(k n)} \times\left(\vec{\lambda}^{(k)} \times \vec{\sigma}^{(k)}\right)\right]_{2} ;
\end{aligned}
$$

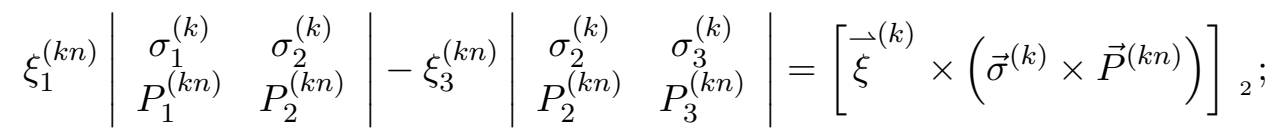

$$
\begin{aligned}
& L_{k n} \xi_{1}^{(k n)}\left|\begin{array}{cc}
\lambda_{1}^{(k)} & \lambda_{2}^{(k)} \\
\sigma_{1}^{(k)} & \sigma_{2}^{(k)}
\end{array}\right|-L_{k n} \xi_{3}^{(k n)}\left|\begin{array}{cc}
\lambda_{2}^{(k)} & \lambda_{3}^{(k)} \\
\sigma_{2}^{(k)} & \sigma_{3}^{(k)}
\end{array}\right|=-L_{k n}\left[\vec{\xi}^{(k)} \times\left(\vec{\lambda}^{(k)} \times \vec{\sigma}^{(k)}\right)\right] 2
\end{aligned}
$$

that is,

$$
\begin{aligned}
-\frac{d \sigma_{2}^{(k)}}{d s_{k}} & =\sum_{n=1, n \neq k}^{3} \frac{e_{k} e_{n}}{m_{k} c^{2}}\left\{-\left[\vec{P}^{(k n)} \times\left(\vec{\sigma}^{(k)} \times \vec{\xi}^{(k)}\right)\right]_{2}-\tau_{k n}\left[\vec{P}^{(k n)} \times\left(\vec{\sigma}^{(k)} \times \vec{\lambda}^{(k)}\right)\right]_{2}+\right. \\
& \left.+\left[\vec{\xi}^{(k)} \times\left(\vec{\sigma}^{(k)} \times \vec{P}^{(k n)}\right)\right]_{2}-L_{k n} L_{k n}\left[\vec{\xi}^{(k)} \times\left(\vec{\lambda}^{(k)} \times \vec{\sigma}^{(k)}\right)\right]_{2}\right\}
\end{aligned}
$$


or

$$
\begin{aligned}
\frac{d \sigma_{2}^{(k)}}{d s_{k}} & =\sum_{n=1, n \neq k}^{3} \frac{e_{k} e_{n}}{m_{k} c^{2}}\left\{\left[\vec{P}^{(k n)} \times\left(\vec{\sigma}^{(k)} \times \vec{\xi}^{(k n)}\right)\right]_{2}+\tau_{k n} \tau_{k n}\left[\vec{P}^{(k n)} \times\left(\vec{\lambda}^{(k)} \times \vec{\sigma}^{(k)}\right)\right]_{2}-\right. \\
- & {\left.\left[\vec{\xi}^{(k n)} \times\left(\vec{\sigma}^{(k)} \times \vec{P}^{(k n)}\right)\right]_{2}+L_{k n} L_{k n}\left[\vec{\xi}^{(k n)} \times\left(\vec{\lambda}^{(k)} \times \vec{\sigma}^{(k)}\right)\right]_{2}\right\} . }
\end{aligned}
$$

Transformation of the Lorentz part of equation 3.3

For the Lorentz part of the third equation

$$
\frac{d \sigma_{23}^{(k)}}{d s_{k}}=\frac{e_{k}}{m_{k} c^{2}} \sum_{n=1, n \neq k}^{3}\left(F_{2 m}^{(k n)} \sigma_{m 3}^{(k)}-F_{m 3}^{(k n)} \sigma_{2 m}^{(k)}\right)+\frac{e_{k}}{m_{k} c^{2}}\left(F_{2 m}^{(k) r a d} \sigma_{m 3}^{(k)}-F_{m 3}^{(k) r a d} \sigma_{2 m}^{(k)}\right)
$$

we have

$$
\begin{aligned}
& \sum_{n=1, n \neq k}^{3}\left(F_{2 m}^{(k n)} \sigma_{m 3}^{(k)}-F_{m 3}^{(k n)} \sigma_{2 m}^{(k)}\right)=\sum_{n=1, n \neq k}^{3} e_{n}\left(\left(P_{2}^{(k n)} \xi_{m}^{(k n)}-P_{m}^{(k n)} \xi_{2}^{(k n)}\right) \sigma_{m 3}^{(k)}-\right. \\
& \left.-\left(P_{m}^{(k n)} \xi_{3}^{(k n)}-P_{3}^{(k n)} \xi_{m}^{(k n)}\right) \sigma_{2 m}^{(k)}\right) \\
& =\sum_{n=1, n \neq k}^{3} e_{n}\left(P_{2}^{(k n)} \sigma_{m 3}^{(k)} \xi_{m}^{(k n)}-\sigma_{m 3}^{(k)} P_{m}^{(k n)} \xi_{2}^{(k n)}-P_{m}^{(k n)} \sigma_{2 m}^{(k)} \xi_{3}^{(k n)}+P_{3}^{(k n)} \sigma_{2 m}^{(k)} \xi_{m}^{(k n)}\right) \text {; } \\
& P_{2}^{(k n)} \sigma_{m 3}^{(k)} \xi_{m}^{(k n)}=P_{2}^{(k n)}\left(\sigma_{13}^{(k)} \xi_{1}^{(k n)}+\sigma_{23}^{(k)} \xi_{2}^{(k n)}+\sigma_{43}^{(k)} \xi_{4}^{(k n)}\right)=P_{2}^{(k n)}\left(-\sigma_{2}^{(k)} \xi_{1}^{(k n)}+\sigma_{1}^{(k)} \xi_{2}^{(k n)}+\sigma_{43}^{(k)} \xi_{4}^{(k n)}\right) \\
& =P_{2}^{(k n)}\left|\begin{array}{cc}
\sigma_{1}^{(k)} & \sigma_{2}^{(k)} \\
\xi_{1}^{(k n)} & \xi_{2}^{(k n)}
\end{array}\right|+\tau_{k n} P_{2}^{(k n)}\left|\begin{array}{cc}
\lambda_{1}^{(k)} & \lambda_{2}^{(k)} \\
\sigma_{1}^{(k)} & \sigma_{2}^{(k)}
\end{array}\right| ; \\
& -\xi_{2}^{(k n)} \sigma_{m 3}^{(k)} P_{m}^{(k n)}=-\xi_{2}^{(k n)}\left(\sigma_{13}^{(k)} P_{1}^{(k n)}+\sigma_{23}^{(k)} P_{2}^{(k n)}+\sigma_{43}^{(k)} P_{4}^{(k n)}\right)=-\xi_{2}^{(k n)}\left(-\sigma_{2}^{(k)} P_{1}^{(k n)}+\sigma_{1}^{(k)} P_{2}^{(k n)}+\sigma_{43}^{(k)} P_{4}^{(k n)}\right) \\
& =-\xi_{2}^{(k n)}\left|\begin{array}{cc}
\sigma_{1}^{(k)} & \sigma_{2}^{(k)} \\
P_{1}^{(k n)} & P_{2}^{(k n)}
\end{array}\right|+L_{k n} \xi_{2}^{(k n)}\left|\begin{array}{cc}
\lambda_{1}^{(k)} & \lambda_{2}^{(k)} \\
\sigma_{1}^{(k)} & \sigma_{2}^{(k)}
\end{array}\right| ; \\
& -\xi_{3}^{(k n)} P_{m}^{(k n)} \sigma_{2 m}^{(k)}=-\xi_{3}^{(k n)}\left(P_{1}^{(k n)} \sigma_{21}^{(k)}+P_{3}^{(k n)} \sigma_{23}^{(k)}+P_{4}^{(k n)} \sigma_{24}^{(k)}\right)=-\xi_{3}^{(k n)}\left(-P_{1}^{(k n)} \sigma_{3}^{(k)}+P_{3}^{(k n)} \sigma_{1}^{(k)}+P_{4}^{(k n)} \sigma_{24}^{(k)}\right) \\
& =-\xi_{3}^{(k n)}\left|\begin{array}{cc}
\sigma_{1}^{(k)} & \sigma_{3}^{(k)} \\
P_{1}^{(k n)} & P_{3}^{(k n)}
\end{array}\right|+L_{k n} \xi_{3}^{(k n)}\left|\begin{array}{cc}
\lambda_{1}^{(k)} & \lambda_{3}^{(k)} \\
\sigma_{1}^{(k)} & \sigma_{3}^{(k)}
\end{array}\right| ; \\
& P_{3}^{(k n)} \sigma_{2 m}^{(k)} \xi_{m}^{(k n)}=P_{3}^{(k n)}\left(\sigma_{21}^{(k)} \xi_{1}^{(k n)}+\sigma_{23}^{(k)} \xi_{3}^{(k n)}+\sigma_{24}^{(k)} \xi_{4}^{(k n)}\right)=P_{3}^{(k n)}\left(-\sigma_{3}^{(k)} \xi_{1}^{(k n)}+\sigma_{1}^{(k)} \xi_{3}^{(k n)}+\sigma_{24}^{(k)} \xi_{4}^{(k n)}\right) \\
& =P_{3}^{(k n)}\left|\begin{array}{cc}
\sigma_{1}^{(k)} & \sigma_{3}^{(k)} \\
\xi_{1}^{(k n)} & \xi_{3}^{(k n)}
\end{array}\right|+\tau_{k n} P_{3}^{(k n)}\left|\begin{array}{cc}
\lambda_{1}^{(k)} & \lambda_{3}^{(k)} \\
\sigma_{1}^{(k)} & \sigma_{3}^{(k)}
\end{array}\right| .
\end{aligned}
$$

Further on we have

$$
\begin{aligned}
& \sum_{n=1, n \neq k}^{3}\left(F_{2 m}^{(k n)} \sigma_{m 3}^{(k)}-F_{m 3}^{(k n)} \sigma_{2 m}^{(k)}\right)=e_{n}\left(P_{2}^{(k n)} \sigma_{m 3}^{(k)} \xi_{m}^{(k n)}-\sigma_{m 3}^{(k)} P_{m}^{(k n)} \xi_{2}^{(k n)}-P_{m}^{(k n)} \sigma_{2 m}^{(k)} \xi_{3}^{(k n)}+P_{3}^{(k n)} \sigma_{2 m}^{(k)} \xi_{m}^{(k n)}\right)=
\end{aligned}
$$

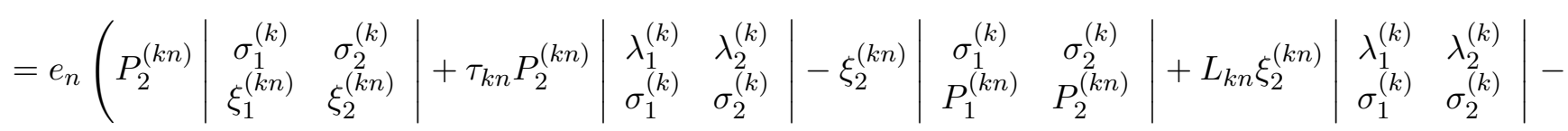

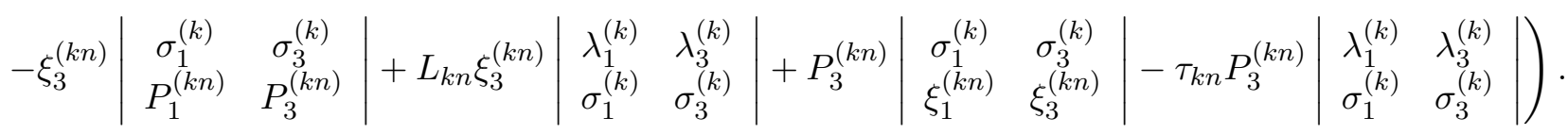


Then

$$
\begin{aligned}
\frac{d \sigma_{1}^{(k)}}{d s_{k}} & =\sum_{\substack{n=1, n \neq k}}^{3} \frac{e_{k} e_{n}}{m_{k} c^{2}}\left(P_{2}^{(k n)}\left|\begin{array}{cc}
\sigma_{1}^{(k)} & \sigma_{2}^{(k)} \\
\xi_{1}^{(k n)} & \xi_{2}^{(k n)}
\end{array}\right|+P_{3}^{(k n)}\left|\begin{array}{cc}
\sigma_{1}^{(k)} & \sigma_{3}^{(k)} \\
\xi_{1}^{(k n)} & \xi_{3}^{(k n)}
\end{array}\right|+\tau_{k n} P_{2}^{(k n)}\left|\begin{array}{cc}
\lambda_{1}^{(k)} & \lambda_{2}^{(k)} \\
\sigma_{1}^{(k)} & \sigma_{2}^{(k)}
\end{array}\right|+\right. \\
& +\tau_{k n} P_{3}^{(k n)}\left|\begin{array}{cc}
\lambda_{1}^{(k)} & \lambda_{3}^{(k)} \\
\sigma_{1}^{(k)} & \sigma_{3}^{(k)}
\end{array}\right|-\xi_{2}^{(k n)}\left|\begin{array}{cc}
\sigma_{1}^{(k)} & \sigma_{2}^{(k)} \\
P_{1}^{(k n)} & P_{2}^{(k n)}
\end{array}\right|-\xi_{3}^{(k n)}\left|\begin{array}{cc}
\sigma_{1}^{(k)} & \sigma_{3}^{(k)} \\
P_{1}^{(k n)} & P_{3}^{(k n)}
\end{array}\right|+ \\
& \left.+L_{k n} \xi_{2}^{(k n)}\left|\begin{array}{cc|c|}
\lambda_{1}^{(k)} & \lambda_{2}^{(k)} \\
\sigma_{1}^{(k)} & \sigma_{2}^{(k)}
\end{array}\right|+L_{k n} \xi_{3}^{(k n)}\left|\begin{array}{ccc}
\lambda_{1}^{(k)} & \lambda_{3}^{(k)} \\
\sigma_{1}^{(k)} & \sigma_{3}^{(k)}
\end{array}\right|\right) .
\end{aligned}
$$

But

$$
\begin{gathered}
P_{2}^{(k n)}\left|\begin{array}{cc}
\sigma_{1}^{(k)} & \sigma_{2}^{(k)} \\
\xi_{1}^{(k n)} & \xi_{2}^{(k n)}
\end{array}\right|+P_{3}^{(k n)}\left|\begin{array}{cc}
\sigma_{1}^{(k)} & \sigma_{3}^{(k)} \\
\xi_{1}^{(k n)} & \xi_{3}^{(k n)}
\end{array}\right|=\left[\vec{P}^{(k n)} \times\left(\vec{\sigma}^{(k)} \times \vec{\xi}^{(k n)}\right)\right]_{1} ; \\
\tau_{k n} P_{2}^{(k n)}\left|\begin{array}{cc}
\lambda_{1}^{(k)} & \lambda_{2}^{(k)} \\
\sigma_{1}^{(k)} & \sigma_{2}^{(k)}
\end{array}\right|+\tau_{k n} P_{3}^{(k n)}\left|\begin{array}{cc}
\lambda_{1}^{(k)} & \lambda_{3}^{(k)} \\
\sigma_{1}^{(k)} & \sigma_{3}^{(k)}
\end{array}\right|=\tau_{k n}\left[\vec{P}^{(k n)} \times\left(\vec{\lambda}^{(k)} \times \vec{\sigma}^{(k)}\right)\right]_{1} ; \\
-\xi_{2}^{(k n)}\left|\begin{array}{cc}
\sigma_{1}^{(k)} & \sigma_{2}^{(k)} \\
P_{1}^{(k n)} & P_{2}^{(k n)}
\end{array}\right|-\xi_{3}^{(k n)}\left|\begin{array}{cc}
\sigma_{1}^{(k)} & \sigma_{3}^{(k)} \\
P_{1}^{(k n)} & P_{3}^{(k n)}
\end{array}\right|=-\left[\vec{\xi}^{(k n)} \times\left(\vec{\sigma}^{(k)} \times \vec{P}^{(k n)}\right)\right]_{1} ; \\
L_{k n} \xi_{2}^{(k n)}\left|\begin{array}{cc}
\lambda_{1}^{(k)} & \lambda_{2}^{(k)} \\
\sigma_{1}^{(k)} & \sigma_{2}^{(k)}
\end{array}\right|+L_{k n} \xi_{3}^{(k n)}\left|\begin{array}{cc}
\lambda_{1}^{(k)} & \lambda_{3}^{(k)} \\
\sigma_{1}^{(k)} & \sigma_{3}^{(k)}
\end{array}\right|=L_{k n}\left[\vec{\xi}^{(k n)} \times\left(\vec{\lambda}^{(k)} \times \vec{\sigma}^{(k)}\right)\right]_{1}
\end{gathered}
$$

and then

$$
\begin{aligned}
\frac{d \sigma_{1}^{(k)}}{d s_{k}}= & \sum_{n=1, n \neq k}^{3} \frac{e_{k} e_{n}}{m_{k} c^{2}}\left\{\left[\vec{P}^{(k n)} \times\left(\vec{\sigma}^{(k)} \times \vec{\xi}^{(k n)}\right)\right]_{1}+\tau_{k n}\left[\vec{P}^{(k n)} \times\left(\vec{\lambda}^{(k)} \times \vec{\sigma}^{(k)}\right)\right]_{1}-\right. \\
& \left.-\left[\vec{\xi}^{(k n)} \times\left(\vec{\sigma}^{(k)} \times \vec{P}^{(k n)}\right)\right]_{1}+L_{k n}\left[\vec{\xi}^{(k n)} \times\left(\vec{\lambda}^{(k)} \times \vec{\sigma}^{(k)}\right)\right]_{1}\right\} .
\end{aligned}
$$

Finally, we rewrite the Lorentz parts of the first three spin equation in a vector form

$$
\begin{aligned}
\frac{d \vec{\sigma}^{(k)}}{d s_{k}}= & \sum_{n=1, n \neq k}^{3} \frac{e_{k} e_{n}}{m_{k} c^{2}}\left[\vec{P}^{(k n)} \times\left(\vec{\sigma}^{(k)} \times \vec{\xi}^{(k n)}\right)+\tau_{k n} \vec{P}^{(k n)} \times\left(\vec{\lambda}^{(k)} \times \vec{\sigma}^{(k)}\right)-\vec{\xi}^{(k n)} \times\left(\vec{\sigma}^{(k)} \times \vec{P}^{(k n)}\right)+\right. \\
& \left.+L_{k n} \vec{\xi}^{(k n)} \times\left(\vec{\lambda}^{(k)} \times \vec{\sigma}^{(k)}\right)\right] .
\end{aligned}
$$

Having in mind that

$$
\begin{aligned}
& \frac{e_{k}}{m_{k} c^{2}}\left(F_{1 m}^{(k) r a d} \sigma_{m 2}^{(k)}-\sigma_{1 m}^{(k)} F_{m 2}^{(k) r a d}\right)=\frac{e_{k}^{2}}{2 m_{k} c^{2}} \\
& \left\{\left[\vec{P}^{(k) r e t} \times\left(\vec{\sigma}^{(k)} \times \vec{\xi}^{(k) r e t}\right)\right]_{3}+\tau_{k}^{r e t}\left[\vec{P}^{(k) r e t} \times\left(\vec{\lambda}^{(k)} \times \vec{\sigma}^{(k)}\right)\right]_{3}-\left[\vec{\xi}^{(k) r e t} \times\left(\vec{\sigma}^{(k)} \times \vec{P}^{(k) r e t}\right)\right]_{3}+\right. \\
& \left.\quad+L_{k, r e t}\left[\vec{\xi}^{(k) r e t} \times\left(\vec{\lambda}^{(k)} \times \vec{\sigma}^{(k)}\right)\right]_{3}\right\}- \\
& -\left\{\left[\vec{P}^{(k) a d v} \times\left(\vec{\sigma}^{(k)} \times \vec{\xi}^{(k) a d v}\right)\right]_{3}+\tau_{k}^{a d v}\left[\vec{P}^{(k) a d v} \times\left(\vec{\lambda}^{(k)} \times \vec{\sigma}^{(k)}\right)\right]_{3}-\left[\vec{\xi}^{(k) a d v} \times\left(\vec{\sigma}^{(k)} \times \vec{P}^{(k) a d v)}+\right.\right.\right. \\
& \left.\quad+L_{k, a d v}\left[\vec{\xi}^{(k) a d v} \times\left(\vec{\lambda}^{(k)} \times \vec{\sigma}^{(k)}\right)\right]_{3}\right\} ;
\end{aligned}
$$




$$
\begin{aligned}
& \frac{e_{k}}{m_{k} c^{2}}\left(F_{1 m}^{(k) r a d} \sigma_{m 3}^{(k)}-\sigma_{1 m}^{(k)} F_{m 3}^{(k) r a d}\right)=\frac{e_{k}^{2}}{2 m_{k} c^{2}} \\
& \left\{\left[\vec{P}^{(k) r e t} \times\left(\vec{\sigma}^{(k)} \times \vec{\xi}^{(k) r e t}\right)\right]_{2}+\tau_{k}^{r e t}\left[\vec{P}^{(k) r e t} \times\left(\vec{\lambda}^{(k)} \times \vec{\sigma}^{(k)}\right)\right]_{2}-\left[\vec{\xi}^{(k) r e t} \times\left(\vec{\sigma}^{(k)} \times \vec{P}^{(k) r e t}\right)\right]_{2}+\right. \\
& \left.+L_{k, \text { ret }}\left[\vec{\xi}^{(k) \text { ret }} \times\left(\vec{\lambda}^{(k)} \times \vec{\sigma}^{(k)}\right)\right]_{2}\right\}-\left\{\left[\vec{P}^{(k) a d v} \times\left(\vec{\sigma}^{(k)} \times \vec{\xi}^{(k) a d v}\right)\right]_{2}+\tau_{k}^{a d v}\left[\vec{P}^{(k) a d v} \times\left(\vec{\lambda}^{(k)} \times \vec{\sigma}^{(k)}\right)\right]_{2}-\right. \\
& \left.-\left[\vec{\xi}^{(k) a d v} \times\left(\vec{\sigma}^{(k)} \times \vec{P}^{(k) a d v}\right)\right]_{2}+L_{k, a d v}\left[\vec{\xi}^{(k) a d v} \times\left(\vec{\lambda}^{(k)} \times \vec{\sigma}^{(k)}\right)\right]_{2}\right\} ; \\
& \frac{e_{k}}{m_{k} c^{2}}\left(F_{2 m}^{(k) r a d} \sigma_{m 3}^{(k)}-F_{m 3}^{(k) r a d} \sigma_{2 m}^{(k)}\right)=\frac{e_{k}^{2}}{2 m_{k} c^{2}} \\
& \left\{\left[\vec{P}^{(k) r e t} \times\left(\vec{\sigma}^{(k)} \times \vec{\xi}^{(k) r e t}\right)\right]_{1}+\tau_{k}^{r e t}\left[\vec{P}^{(k) r e t} \times\left(\vec{\lambda}^{(k)} \times \vec{\sigma}^{(k)}\right)\right]_{1}-\left[\vec{\xi}^{(k) r e t} \times\left(\vec{\sigma}^{(k)} \times \vec{P}^{(k) r e t}\right)\right]_{1}+\right. \\
& \left.+L_{k, r e t}\left[\vec{\xi}^{(k) \text { ret }} \times\left(\vec{\lambda}^{(k)} \times \vec{\sigma}^{(k)}\right)\right]_{1}\right\}- \\
& -\left\{\left[\vec{P}^{(k) a d v} \times\left(\vec{\sigma}^{(k)} \times \vec{\xi}^{(k) a d v}\right)\right]_{1}+\tau_{k}^{a d v}\left[\vec{P}^{(k) a d v} \times\left(\vec{\lambda}^{(k)} \times \vec{\sigma}^{(k)}\right)\right]_{1}-\left[\vec{\xi}^{(k) a d v} \times\left(\vec{\sigma}^{(k)} \times \vec{P}^{(k) a d v}\right)\right]_{1}+\right. \\
& \left.+L_{k, a d v}\left[\vec{\xi}^{(k) a d v} \times\left(\vec{\lambda}^{(k)} \times \vec{\sigma}^{(k)}\right)\right]_{1}\right\}
\end{aligned}
$$

we obtain

$$
\begin{aligned}
\frac{d \vec{\sigma}^{(k)}}{d s_{k}}= & \sum_{n=1, n \neq k}^{3} \frac{e_{k} e_{n}}{m_{k} c^{2}}\left[\vec{P}^{(k n)} \times\left(\vec{\sigma}^{(k)} \times \vec{\xi}^{(k n)}\right)+\tau_{k n} \vec{P}^{(k n)} \times\left(\vec{\lambda}^{(k)} \times \vec{\sigma}^{(k)}\right)-\vec{\xi}^{(k n)} \times\left(\vec{\sigma}^{(k)} \times \vec{P}^{(k n)}\right)+\right. \\
& \left.+L_{k n} \vec{\xi}^{(k n)} \times\left(\vec{\lambda}^{(k)} \times \vec{\sigma}^{(k)}\right)\right]+\frac{e_{k}{ }^{2}}{2 m_{k} c^{2}}\left\{\left[\vec{P}^{(k) r e t} \times\left(\vec{\sigma}^{(k)} \times \vec{\xi}^{(k) r e t}\right)+\tau_{k}^{r e t} \vec{P}^{(k) r e t} \times\left(\vec{\lambda}^{(k)} \times \vec{\sigma}^{(k)}\right)-\right.\right. \\
& \left.-\vec{\xi}^{(k) r e t} \times\left(\vec{\sigma}^{(k)} \times \vec{P}^{(k) r e t}\right)+L_{k, r e t} \vec{\xi}^{(k) r e t} \times\left(\vec{\lambda}^{(k)} \times \vec{\sigma}^{(k)}\right)\right]- \\
& -\left[\vec{P}^{(k) a d v} \times\left(\vec{\sigma}^{(k)} \times \vec{\xi}^{(k) a d v}\right)+\tau_{k}^{a d v} \vec{P}^{(k) a d v} \times\left(\vec{\lambda}^{(k)} \times \vec{\sigma}^{(k)}\right)-\vec{\xi}^{(k) a d v} \times\left(\vec{\sigma}^{(k)} \times \vec{P}^{(k) a d v}\right)+\right. \\
& \left.\left.+L_{k, a d v} \vec{\xi}^{(k) a d v} \times\left(\vec{\lambda}^{(k)} \times \vec{\sigma}^{(k)}\right)\right]\right\} .
\end{aligned}
$$

Transformation of the spin equation 3.4

$$
\begin{aligned}
& \frac{d \sigma_{14}^{(k)}}{d s_{k}}=\frac{e_{k}}{m_{k} c^{2}}\left[\sum_{n=1, n \neq k}^{3}\left(F_{1 m}^{(k n)} \sigma_{m 4}^{(k)}-F_{m 4}^{(k n)} \sigma_{1 m}^{(k)}\right)+F_{1 m}^{(k) \text { rad }} \sigma_{m 4}^{(k)}-F_{m 4}^{(k) r a d} \sigma_{1 m}^{(k)}\right] ; \\
& F_{1 m}^{(k n)} \sigma_{m 4}^{(k)}-F_{m 4}^{(k n)} \sigma_{1 m}^{(k)}=e_{n}\left(P_{1}^{(k n)} \xi_{m}^{(k n)} \sigma_{m 4}^{(k)}-P_{m}^{(k n)} \sigma_{m 4}^{(k)} \xi_{1}^{(k n)}-P_{m}^{(k n)} \sigma_{1 m}^{(k)} \xi_{4}^{(k n)}+P_{4}^{(k n)} \sigma_{1 m}^{(k)} \xi_{m}^{(k n)}\right) \\
& P_{1}^{(k n)} \xi_{m}^{(k n)} \sigma_{m 4}^{(k)}=P_{1}^{(k n)}\left(\xi_{1}^{(k n)} \sigma_{14}^{(k)}+\xi_{2}^{(k n)} \sigma_{24}^{(k)}+\xi_{3}^{(k n)} \sigma_{34}^{(k)}\right) \\
& =P_{1}^{(k n)}\left(\xi_{1}^{(k n)} \frac{i}{c}\left|\begin{array}{cc}
\lambda_{2}^{(k)} & \lambda_{3}^{(k)} \\
\sigma_{2}^{(k)} & \sigma_{3}^{(k)}
\end{array}\right|-\xi_{2}^{(k n)} \frac{i}{c}\left|\begin{array}{cc}
\lambda_{1}^{(k)} & \lambda_{3}^{(k)} \\
\sigma_{1}^{(k)} & \sigma_{3}^{(k)}
\end{array}\right|+\xi_{3}^{(k n)} \frac{i}{c}\left|\begin{array}{cc}
\lambda_{1}^{(k)} & \lambda_{2}^{(k)} \\
\sigma_{1}^{(k)} & \sigma_{2}^{(k)}
\end{array}\right|\right) \\
& =\frac{i P_{1}^{(k n)}}{c}\left|\begin{array}{ccc}
\xi_{1}^{(k n)} & \xi_{2}^{(k n)} & \xi_{3}^{(k n)} \\
\lambda_{1}^{(k)} & \lambda_{2}^{(k)} & \lambda_{3}^{(k)} \\
\sigma_{1}^{(k)} & \sigma_{2}^{(k)} & \sigma_{3}^{(k)}
\end{array}\right|
\end{aligned}
$$




$$
\begin{aligned}
& -\xi_{1}^{(k n)} P_{m}^{(k n)} \sigma_{m 4}^{(k)}=-\xi_{1}^{(k n)}\left(P_{1}^{(k n)} \sigma_{14}^{(k)}+P_{2}^{(k n)} \sigma_{24}^{(k)}+P_{3}^{(k n)} \sigma_{34}^{(k)}\right) \\
& =-\xi_{1}^{(k n)}\left(\frac{i P_{1}^{(k n)}}{c}\left|\begin{array}{cc}
\lambda_{2}^{(k)} & \lambda_{3}^{(k)} \\
\sigma_{2}^{(k)} & \sigma_{3}^{(k)}
\end{array}\right|-\frac{i P_{2}^{(k n)}}{c}\left|\begin{array}{cc}
\lambda_{1}^{(k)} & \lambda_{3}^{(k)} \\
\sigma_{1}^{(k)} & \sigma_{3}^{(k)}
\end{array}\right|+\frac{i P_{3}^{(k n)}}{c}\left|\begin{array}{cc}
\lambda_{1}^{(k)} & \lambda_{2}^{(k)} \\
\sigma_{1}^{(k)} & \sigma_{2}^{(k)}
\end{array}\right|\right) \\
& =-\frac{i \xi_{1}^{(k n)}}{c}\left|\begin{array}{ccc}
P_{1}^{(k n)} & P_{2}^{(k n)} & P_{3}^{(k n)} \\
\lambda_{1}^{(k)} & \lambda_{2}^{(k)} & \lambda_{3}^{(k)} \\
\sigma_{1}^{(k)} & \sigma_{2}^{(k)} & \sigma_{3}^{(k)}
\end{array}\right| \\
& -\xi_{4}^{(k n)} P_{m}^{(k n)} \sigma_{1 m}^{(k)}=-\xi_{4}^{(k n)}\left(P_{2}^{(k n)} \sigma_{12}^{(k)}+P_{3}^{(k n)} \sigma_{13}^{(k)}+P_{4}^{(k n)} \sigma_{14}^{(k)}\right) \\
& =-\xi_{4}^{(k n)}\left(P_{2}^{(k n)} \sigma_{3}^{(k)}-P_{3}^{(k n)} \sigma_{2}^{(k)}+P_{4}^{(k n)} \sigma_{14}^{(k)}\right) \\
& =-\xi_{4}^{(k n)}\left(-\left|\begin{array}{cc}
\sigma_{2}^{(k)} & \sigma_{3}^{(k)} \\
P_{2}^{(k n)} & P_{3}^{(k n)}
\end{array}\right|+\frac{i P_{4}^{(k n)}}{c}\left|\begin{array}{cc}
\lambda_{2}^{(k)} & \lambda_{3}^{(k)} \\
\sigma_{2}^{(k)} & \sigma_{3}^{(k)}
\end{array}\right|\right) \\
& =i c \tau_{k n}\left|\begin{array}{cc}
\sigma_{2}^{(k)} & \sigma_{3}^{(k)} \\
P_{2}^{(k n)} & P_{3}^{(k n)}
\end{array}\right|-i c \tau_{k n} L_{k n}\left|\begin{array}{cc}
\lambda_{2}^{(k)} & \lambda_{3}^{(k)} \\
\sigma_{2}^{(k)} & \sigma_{3}^{(k)}
\end{array}\right| \text {; } \\
& P_{4}^{(k n)} \sigma_{1 m}^{(k)} \xi_{m}^{(k n)}=P_{4}^{(k n)}\left(\sigma_{12}^{(k)} \xi_{2}^{(k n)}+\sigma_{13}^{(k)} \xi_{3}^{(k n)}+\sigma_{14}^{(k)} \xi_{4}^{(k n)}\right) \\
& =P_{4}^{(k n)}\left(\sigma_{3}^{(k)} \xi_{2}^{(k n)}-\sigma_{2}^{(k)} \xi_{3}^{(k n)}+\sigma_{14}^{(k)} \xi_{4}^{(k n)}\right) \\
& =-P_{4}^{(k n)}\left|\begin{array}{cc}
\sigma_{2}^{(k)} & \sigma_{3}^{(k)} \\
\xi_{2}^{(k n)} & \xi_{3}^{(k n)}
\end{array}\right|+P_{4}^{(k n)} i c \tau_{k n} \frac{i}{c}\left|\begin{array}{cc}
\lambda_{2}^{(k)} & \lambda_{3}^{(k)} \\
\sigma_{2}^{(k)} & \sigma_{3}^{(k)}
\end{array}\right| \\
& =i c L_{k n}\left|\begin{array}{cc}
\sigma_{2}^{(k)} & \sigma_{3}^{(k)} \\
\xi_{2}^{(k n)} & \xi_{3}^{(k n)}
\end{array}\right|+i c L_{k n} \tau_{k n}\left|\begin{array}{cc}
\lambda_{2}^{(k)} & \lambda_{3}^{(k)} \\
\sigma_{2}^{(k)} & \sigma_{3}^{(k)}
\end{array}\right| \text {. }
\end{aligned}
$$

Then

$$
\begin{aligned}
& F_{1 m}^{(k n)} \sigma_{m 4}^{(k)}-F_{m 4}^{(k n)} \sigma_{1 m}^{(k)}=e_{n}\left(\frac{i P_{1}^{(k n)}}{c}\left|\begin{array}{ccc}
\xi_{1}^{(k n)} & \xi_{2}^{(k n)} & \xi_{3}^{(k n)} \\
\lambda_{1}^{(k)} & \lambda_{2}^{(k)} & \lambda_{3}^{(k)} \\
\sigma_{1}^{(k)} & \sigma_{2}^{(k)} & \sigma_{3}^{(k)}
\end{array}\right|-\frac{i \xi_{1}^{(k n)}}{c}\left|\begin{array}{ccc}
P_{1}^{(k n)} & P_{2}^{(k n)} & P_{3}^{(k n)} \\
\lambda_{1}^{(k)} & \lambda_{2}^{(k)} & \lambda_{3}^{(k)} \\
\sigma_{1}^{(k)} & \sigma_{2}^{(k)} & \sigma_{3}^{(k)}
\end{array}\right|+\right. \\
& +i c \tau_{k n}\left|\begin{array}{cc}
\sigma_{2}^{(k)} & \sigma_{3}^{(k)} \\
P_{2}^{(k n)} & P_{3}^{(k n)}
\end{array}\right|-i c \tau_{k n} L_{k n}\left|\begin{array}{cc}
\lambda_{2}^{(k)} & \lambda_{3}^{(k)} \\
\sigma_{2}^{(k)} & \sigma_{3}^{(k)}
\end{array}\right|+i c L_{k n}\left|\begin{array}{cc}
\sigma_{2}^{(k)} & \sigma_{3}^{(k)} \\
\xi_{2}^{(k n)} & \xi_{3}^{(k n)}
\end{array}\right| \\
& \left.+i c L_{k n} \tau_{k n}\left|\begin{array}{cc}
\lambda_{2}^{(k)} & \lambda_{3}^{(k)} \\
\sigma_{2}^{(k)} & \sigma_{3}^{(k)}
\end{array}\right|\right) \\
& =e_{n}\left(\frac{i P_{1}^{(k n)}}{c}\left|\begin{array}{ccc}
\xi_{1}^{(k n)} & \xi_{2}^{(k n)} & \xi_{3}^{(k n)} \\
\lambda_{1}^{(k)} & \lambda_{2}^{(k)} & \lambda_{3}^{(k)} \\
\sigma_{1}^{(k)} & \sigma_{2}^{(k)} & \sigma_{3}^{(k)}
\end{array}\right|-\frac{i \xi_{1}^{(k n)}}{c}\left|\begin{array}{ccc}
P_{1}^{(k n)} & P_{2}^{(k n)} & P_{3}^{(k n)} \\
\lambda_{1}^{(k)} & \lambda_{2}^{(k)} & \lambda_{3}^{(k)} \\
\sigma_{1}^{(k)} & \sigma_{2}^{(k)} & \sigma_{3}^{(k)}
\end{array}\right|\right. \\
& \left.+i c \tau_{k n}\left|\begin{array}{cc}
\sigma_{2}^{(k)} & \sigma_{3}^{(k)} \\
P_{2}^{(k n)} & P_{3}^{(k n)}
\end{array}\right|+i c L_{k n}\left|\begin{array}{cc}
\sigma_{2}^{(k)} & \sigma_{3}^{(k)} \\
\xi_{2}^{(k n)} & \xi_{3}^{(k n)}
\end{array}\right|\right)
\end{aligned}
$$




$$
\begin{aligned}
& \frac{i}{c} \frac{d(\vec{\lambda} \times \vec{\sigma})_{1}}{d s_{k}}= \\
& =\sum_{n=1, n \neq k}^{3} \frac{e_{k} e_{n}}{m_{k} c^{2}}\left(\frac{i P_{1}^{(k n)}}{c}\left|\begin{array}{ccc}
\xi_{1}^{(k n)} & \xi_{2}^{(k n)} & \xi_{3}^{(k n)} \\
\lambda_{1}^{(k)} & \lambda_{2}^{(k)} & \lambda_{3}^{(k)} \\
\sigma_{1}^{(k)} & \sigma_{2}^{(k)} & \sigma_{3}^{(k)}
\end{array}\right|-\frac{i \xi_{1}^{(k n)}}{c}\left|\begin{array}{ccc}
P_{1}^{(k n)} & P_{2}^{(k n)} & P_{3}^{(k n)} \\
\lambda_{1}^{(k)} & \lambda_{2}^{(k)} & \lambda_{3}^{(k)} \\
\sigma_{1}^{(k)} & \sigma_{2}^{(k)} & \sigma_{3}^{(k)}
\end{array}\right|+\right. \\
& \left.+i c \tau_{k n}\left|\begin{array}{cc}
\sigma_{2}^{(k)} & \sigma_{3}^{(k)} \\
P_{2}^{(k n)} & P_{3}^{(k n)}
\end{array}\right|+i c L_{k n}\left|\begin{array}{cc}
\sigma_{2}^{(k)} & \sigma_{3}^{(k)} \\
\xi_{2}^{(k n)} & \xi_{3}^{(k n)}
\end{array}\right|\right)
\end{aligned}
$$

Transformation of the spin equation 3.5

$$
\begin{aligned}
& \frac{d \sigma_{24}^{(k)}}{d s_{k}}=\frac{e_{k}}{m_{k} c^{2}}\left[\sum_{n=1, n \neq k}^{3}\left(F_{2 m}^{(k n)} \sigma_{m 4}^{(k)}-F_{m 4}^{(k n)} \sigma_{2 m}^{(k)}\right)+F_{2 m}^{(k) r a d} \sigma_{m 4}^{(k)}-F_{m 4}^{(k) r a d} \sigma_{2 m}^{(k)}\right] \text {; } \\
& F_{2 m}^{(k n)} \sigma_{m 4}^{(k)}-F_{m 4}^{(k n)} \sigma_{2 m}^{(k)}=e_{n}\left(P_{2}^{(k n)} \xi_{m}^{(k n)} \sigma_{m 4}^{(k)}-P_{m}^{(k n)} \sigma_{m 4}^{(k)} \xi_{2}^{(k n)}-P_{m}^{(k n)} \sigma_{2 m}^{(k)} \xi_{4}^{(k n)}+P_{4}^{(k n)} \xi_{m}^{(k n)} \sigma_{2 m}^{(k)}\right) \\
& P_{2}^{(k n)} \xi_{m}^{(k n)} \sigma_{m 4}^{(k)}=P_{2}^{(k n)}\left(\xi_{1}^{(k n)} \sigma_{14}^{(k)}+\xi_{2}^{(k n)} \sigma_{24}^{(k)}+\xi_{3}^{(k n)} \sigma_{34}^{(k)}\right) \\
& =P_{2}^{(k n)}\left(\frac{i \xi_{1}^{(k n)}}{c}\left|\begin{array}{cc}
\lambda_{2}^{(k)} & \lambda_{3}^{(k)} \\
\sigma_{2}^{(k)} & \sigma_{3}^{(k)}
\end{array}\right|-\frac{i \xi_{2}^{(k n)}}{c}\left|\begin{array}{cc}
\lambda_{1}^{(k)} & \lambda_{3}^{(k)} \\
\sigma_{1}^{(k)} & \sigma_{3}^{(k)}
\end{array}\right|+\frac{i \xi_{3}^{(k n)}}{c}\left|\begin{array}{cc}
\lambda_{1}^{(k)} & \lambda_{2}^{(k)} \\
\sigma_{1}^{(k)} & \sigma_{2}^{(k)}
\end{array}\right|\right) \\
& =\frac{i P_{2}^{(k n)}}{c}\left|\begin{array}{ccc}
\xi_{1}^{(k n)} & \xi_{2}^{(k n)} & \xi_{3}^{(k n)} \\
\lambda_{1}^{(k)} & \lambda_{2}^{(k)} & \lambda_{3}^{(k)} \\
\sigma_{1}^{(k)} & \sigma_{2}^{(k)} & \sigma_{3}^{(k)}
\end{array}\right| \\
& \xi_{2}^{(k n)} P_{m}^{(k n)} \sigma_{m 4}^{(k)}=\xi_{2}^{(k n)}\left(P_{1}^{(k n)} \sigma_{14}^{(k)}+P_{2}^{(k n)} \sigma_{24}^{(k)}+P_{3}^{(k n)} \sigma_{34}^{(k)}\right) \\
& =\xi_{2}^{(k n)}\left(\frac{i P_{1}^{(k n)}}{c}\left|\begin{array}{cc}
\sigma_{2}^{(k)} & \sigma_{3}^{(k)} \\
\lambda_{2}^{(k)} & \lambda_{3}^{(k)}
\end{array}\right|-\frac{i P_{2}^{(k n)}}{c}\left|\begin{array}{cc}
\sigma_{1}^{(k)} & \sigma_{3}^{(k)} \\
\lambda_{1}^{(k)} & \lambda_{3}^{(k)}
\end{array}\right|+\frac{i P_{3}^{(k n)}}{c}\left|\begin{array}{cc}
\sigma_{1}^{(k)} & \sigma_{2}^{(k)} \\
\lambda_{1}^{(k)} & \lambda_{2}^{(k)}
\end{array}\right|\right) \\
& =\frac{i}{c} \xi_{2}^{(k n)}\left|\begin{array}{ccc}
P_{1}^{(k n)} & P_{2}^{(k n)} & P_{3}^{(k n)} \\
\lambda_{1}^{(k)} & \lambda_{2}^{(k)} & \lambda_{3}^{(k)} \\
\sigma_{1}^{(k)} & \sigma_{2}^{(k)} & \sigma_{3}^{(k)}
\end{array}\right| \\
& \xi_{4}^{(k n)} P_{m}^{(k n)} \sigma_{2 m}^{(k)}=\xi_{4}^{(k n)}\left(P_{1}^{(k n)} \sigma_{21}^{(k)}+P_{3}^{(k n)} \sigma_{23}^{(k)}+P_{4}^{(k n)} \sigma_{24}^{(k)}\right) \\
& =\xi_{4}^{(k n)}\left(\left|\begin{array}{cc}
\sigma_{1}^{(k)} & \sigma_{3}^{(k)} \\
P_{1}^{(k n)} & P_{3}^{(k n)}
\end{array}\right|-\frac{i P_{4}^{(k n)}}{c}\left|\begin{array}{cc}
\lambda_{1}^{(k)} & \lambda_{3}^{(k)} \\
\sigma_{1}^{(k)} & \sigma_{3}^{(k)}
\end{array}\right|\right) \\
& =\xi_{4}^{(k n)}\left(\left|\begin{array}{cc}
\sigma_{1}^{(k)} & \sigma_{3}^{(k)} \\
P_{1}^{(k n)} & P_{3}^{(k n)}
\end{array}\right|-L_{k n}\left|\begin{array}{cc}
\lambda_{1}^{(k)} & \lambda_{3}^{(k)} \\
\sigma_{1}^{(k)} & \sigma_{3}^{(k)}
\end{array}\right|\right) \\
& P_{4}^{(k n)} \xi_{m}^{(k n)} \sigma_{2 m}^{(k)}=P_{4}^{(k n)}\left(\xi_{1}^{(k n)} \sigma_{21}^{(k)}+\xi_{3}^{(k n)} \sigma_{23}^{(k)}+\xi_{4}^{(k n)} \sigma_{24}^{(k)}\right) \\
& =P_{4}^{(k n)}\left(\left|\begin{array}{cc}
\sigma_{1}^{(k)} & \sigma_{3}^{(k)} \\
\xi_{1}^{(k n)} & \xi_{3}^{(k n)}
\end{array}\right|-\frac{i}{c} \xi_{4}^{(k n)}\left|\begin{array}{cc}
\lambda_{1}^{(k)} & \lambda_{3}^{(k)} \\
\sigma_{1}^{(k)} & \sigma_{3}^{(k)}
\end{array}\right|\right) \\
& =P_{4}^{(k n)}\left(\left|\begin{array}{cc}
\sigma_{1}^{(k)} & \sigma_{3}^{(k)} \\
\xi_{1}^{(k n)} & \xi_{3}^{(k n)}
\end{array}\right|+\tau_{k n}\left|\begin{array}{cc}
\lambda_{1}^{(k)} & \lambda_{3}^{(k)} \\
\sigma_{1}^{(k)} & \sigma_{3}^{(k)}
\end{array}\right|\right) \text {; }
\end{aligned}
$$




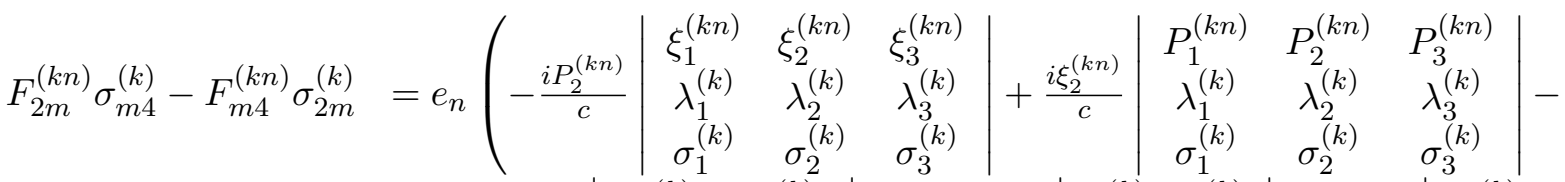

$$
\begin{aligned}
& -i c \tau_{k n}\left|\begin{array}{cc}
\sigma_{1}^{(k)} & \sigma_{3}^{(k)} \\
P_{1}^{(k n)} & P_{3}^{(k n)}
\end{array}\right|-i c \tau_{k n} L_{k n}\left|\begin{array}{cc}
\lambda_{1}^{(k)} & \lambda_{3}^{(k)} \\
\sigma_{1}^{(k)} & \sigma_{3}^{(k)}
\end{array}\right|-i c L_{k n}\left|\begin{array}{cc}
\sigma_{1}^{(k)} & \sigma_{3}^{(k)} \\
\xi_{1}^{(k n)} & \xi_{3}^{(k n)}
\end{array}\right|+ \\
& \left.+i c \tau_{k n} L_{k n}\left|\begin{array}{cc}
\lambda_{1}^{(k)} & \lambda_{3}^{(k)} \\
\sigma_{1}^{(k)} & \sigma_{3}^{(k)}
\end{array}\right|\right) \\
& =e_{n}\left(-\frac{i P_{2}^{(k n)}}{c}\left|\begin{array}{ccc}
\xi_{1}^{(k n)} & \xi_{2}^{(k n)} & \xi_{3}^{(k n)} \\
\lambda_{1}^{(k)} & \lambda_{2}^{(k)} & \lambda_{3}^{(k)} \\
\sigma_{1}^{(k)} & \sigma_{2}^{(k)} & \sigma_{3}^{(k)}
\end{array}\right|+\frac{i \xi_{2}^{(k n)}}{c}\left|\begin{array}{ccc}
P_{1}^{(k n)} & P_{2}^{(k n)} & P_{3}^{(k n)} \\
\lambda_{1}^{(k)} & \lambda_{2}^{(k)} & \lambda_{3}^{(k)} \\
\sigma_{1}^{(k)} & \sigma_{2}^{(k)} & \sigma_{3}^{(k)}
\end{array}\right|-\right. \\
& \left.-i c \tau_{k n}\left|\begin{array}{cc}
\sigma_{1}^{(k)} & \sigma_{3}^{(k)} \\
P_{1}^{(k n)} & P_{3}^{(k n)}
\end{array}\right|-i c L_{k n}\left|\begin{array}{cc}
\sigma_{1}^{(k)} & \sigma_{3}^{(k)} \\
\xi_{1}^{(k n)} & \xi_{3}^{(k n)}
\end{array}\right|\right)
\end{aligned}
$$

or

$$
\begin{aligned}
& -\frac{i}{c} \frac{d}{\Delta_{k} d t}\left|\begin{array}{cc}
\sigma_{1}^{(k)} & \sigma_{3}^{(k)} \\
u_{1}^{(k)} & u_{3}^{(k)}
\end{array}\right|=\sum_{n=1, n \neq k}^{3} \frac{e_{k} e_{n}}{m_{k} c^{2}}\left(-\frac{i P_{2}^{(k n)}}{c}\left|\begin{array}{ccc}
\xi_{1}^{(k n)} & \xi_{2}^{(k n)} & \xi_{3}^{(k n)} \\
\lambda_{1}^{(k)} & \lambda_{2}^{(k)} & \lambda_{3}^{(k)} \\
\sigma_{1}^{(k)} & \sigma_{2}^{(k)} & \sigma_{3}^{(k)}
\end{array}\right|+\frac{i \xi_{2}^{(k n)}}{c}\left|\begin{array}{ccc}
P_{1}^{(k n)} & P_{2}^{(k n)} & P_{3}^{(k n)} \\
\lambda_{1}^{(k)} & \lambda_{2}^{(k)} & \lambda_{3}^{(k)} \\
\sigma_{1}^{(k)} & \sigma_{2}^{(k)} & \sigma_{3}^{(k)}
\end{array}\right|-\right. \\
& \left.-i c \tau_{k n}\left|\begin{array}{cc}
\sigma_{1}^{(k)} & \sigma_{3}^{(k)} \\
P_{1}^{(k n)} & P_{3}^{(k n)}
\end{array}\right|-i c L_{k n}\left|\begin{array}{cc}
\sigma_{1}^{(k)} & \sigma_{3}^{(k)} \\
\xi_{1}^{(k n)} & \xi_{3}^{(k n)}
\end{array}\right|\right) \\
& \frac{i}{c} \frac{d(\vec{\lambda} \times \vec{\sigma})_{2}}{d s_{k}}=\sum_{n=1, n \neq k}^{3} \frac{e_{k} e_{n}}{m_{k} c^{2}}\left(\frac{i P_{2}^{(k n)}}{c}\left|\begin{array}{ccc}
\xi_{1}^{(k n)} & \xi_{2}^{(k n)} & \xi_{3}^{(k n)} \\
\lambda_{1}^{(k)} & \lambda_{2}^{(k)} & \lambda_{3}^{(k)} \\
\sigma_{1}^{(k)} & \sigma_{2}^{(k)} & \sigma_{3}^{(k)}
\end{array}\right|-\frac{i \xi_{2}^{(k n)}}{c}\left|\begin{array}{ccc}
P_{1}^{(k n)} & P_{2}^{(k n)} & P_{3}^{(k n)} \\
\lambda_{1}^{(k)} & \lambda_{2}^{(k)} & \lambda_{3}^{(k)} \\
\sigma_{1}^{(k)} & \sigma_{2}^{(k)} & \sigma_{3}^{(k)}
\end{array}\right|+\right. \\
& \left.+i c \tau_{k n}\left|\begin{array}{cc}
\sigma_{1}^{(k)} & \sigma_{3}^{(k)} \\
P_{1}^{(k n)} & P_{3}^{(k n)}
\end{array}\right|+i c L_{k n}\left|\begin{array}{cc}
\sigma_{1}^{(k)} & \sigma_{3}^{(k)} \\
\xi_{1}^{(k n)} & \xi_{3}^{(k n)}
\end{array}\right|\right) \text {. }
\end{aligned}
$$

Transformation of the spin equation 3.6

$$
\begin{gathered}
\frac{d \sigma_{34}^{(k)}}{d s_{k}}=\frac{e_{k}}{m_{k} c^{2}}\left[\sum_{n=1, n \neq k}^{3}\left(F_{3 m}^{(k n)} \sigma_{m 4}^{(k)}-F_{m 4}^{(k n)} \sigma_{3 m}^{(k)}\right)+F_{3 m}^{(k) r a d} \sigma_{m 4}^{(k)}-F_{m 4}^{(k) r a d} \sigma_{3 m}^{(k)}\right] ; \\
F_{3 m}^{(k n)} \sigma_{m 4}^{(k)}-F_{m 4}^{(k n)} \sigma_{3 m}^{(k)}=e_{n}\left(P_{3}^{(k n)} \xi_{m}^{(k n)} \sigma_{m 4}^{(k)}-P_{m}^{(k n)} \sigma_{m 4}^{(k)} \xi_{3}^{(k n)}-P_{m}^{(k n)} \sigma_{3 m}^{(k)} \xi_{4}^{(k n)}+P_{4}^{(k n)} \xi_{m}^{(k n)} \sigma_{3 m}^{(k)}\right) ; \\
P_{3}^{(k n)} \xi_{m}^{(k n)} \sigma_{m 4}^{(k)}=P_{3}^{(k n)}\left(\xi_{1}^{(k n)} \sigma_{14}^{(k)}+\xi_{2}^{(k n)} \sigma_{24}^{(k)}+\xi_{3}^{(k n)} \sigma_{34}^{(k)}\right)=\frac{i}{c} P_{3}^{(k n)}\left|\begin{array}{ccc}
\xi_{1}^{(k n)} & \xi_{2}^{(k n)} & \xi_{3}^{(k n)} \\
\lambda_{1}^{(k)} & \lambda_{2}^{(k)} & \lambda_{3}^{(k)} \\
\sigma_{1}^{(k)} & \sigma_{2}^{(k)} & \sigma_{3}^{(k)}
\end{array}\right| ; \\
P_{m}^{(k n)} \sigma_{m 4}^{(k)} \xi_{3}^{(k n)}=\left(P_{1}^{(k n)} \sigma_{14}^{(k)}+P_{2}^{(k n)} \sigma_{24}^{(k)}+P_{3}^{(k n)} \sigma_{34}^{(k)}\right) \xi_{3}^{(k n)}=\frac{i}{c} \xi_{3}^{(k n)}\left|\begin{array}{ccc}
P_{1}^{(k n)} & P_{2}^{(k n)} & P_{3}^{(k n)} \\
\lambda_{1}^{(k)} & \lambda_{2}^{(k)} & \lambda_{3}^{(k)} \\
\sigma_{1}^{(k)} & \sigma_{2}^{(k)} & \sigma_{3}^{(k)}
\end{array}\right| ;
\end{gathered}
$$




$$
\begin{aligned}
\xi_{4}^{(k n)} P_{m}^{(k n)} \sigma_{3 m}^{(k)} & =\xi_{4}^{(k n)}\left(P_{1}^{(k n)} \sigma_{31}^{(k)}+P_{2}^{(k n)} \sigma_{32}^{(k)}+P_{4}^{(k n)} \sigma_{34}^{(k)}\right) \\
& =\xi_{4}^{(k n)}\left(-i c \tau_{k n}\left|\begin{array}{cc|c}
\sigma_{1}^{(k)} & \sigma_{2}^{(k)} \\
P_{1}^{(k n)} & P_{2}^{(k n)}
\end{array}\right|+i c \tau_{k n} L_{k n}\left|\begin{array}{cc}
\lambda_{1}^{(k)} & \lambda_{2}^{(k)} \\
\sigma_{1}^{(k)} & \sigma_{2}^{(k)}
\end{array}\right|\right) \\
P_{4}^{(k n)} \xi_{m}^{(k n)} \sigma_{3 m}^{(k)} & =P_{4}^{(k n)}\left(\xi_{1}^{(k n)} \sigma_{31}^{(k)}+\xi_{2}^{(k n)} \sigma_{32}^{(k)}+\xi_{4}^{(k n)} \sigma_{34}^{(k)}\right) \\
& =P_{4}^{(k n)}\left(i c L_{k n}\left|\begin{array}{cc|c}
\sigma_{1}^{(k)} & \sigma_{2}^{(k)} \\
\xi_{1}^{(k n)} & \xi_{2}^{(k n)}
\end{array}\right|-i c \tau_{k n} L_{k n}\left|\begin{array}{cc}
\lambda_{1}^{(k)} & \lambda_{2}^{(k)} \\
\sigma_{1}^{(k)} & \sigma_{2}^{(k)}
\end{array}\right|\right)
\end{aligned}
$$

or

$$
\begin{aligned}
\frac{i}{c} \frac{d(\vec{\lambda} \times \vec{\sigma})_{3}}{d s_{k}}= & \sum_{n=1, n \neq k}^{3} \frac{e_{k} e_{n}}{m_{k} c^{2}}\left(\begin{array}{l}
i \\
c
\end{array} P_{3}^{(k n)}\left|\begin{array}{ccc}
\xi_{1}^{(k n)} & \xi_{2}^{(k n)} & \xi_{3}^{(k n)} \\
\lambda_{1}^{(k)} & \lambda_{2}^{(k)} & \lambda_{3}^{(k)} \\
\sigma_{1}^{(k)} & \sigma_{2}^{(k)} & \sigma_{3}^{(k)}
\end{array}\right|-\frac{i}{c} \xi_{3}^{(k n)}\left|\begin{array}{ccc}
P_{1}^{(k n)} & P_{2}^{(k n)} & P_{3}^{(k n)} \\
\lambda_{1}^{(k)} & \lambda_{2}^{(k)} & \lambda_{3}^{(k)} \\
\sigma_{1}^{(k)} & \sigma_{2}^{(k)} & \sigma_{3}^{(k)}
\end{array}\right|\right. \\
& \left.+i c \tau_{k n}\left|\begin{array}{cc}
\sigma_{1}^{(k)} & \sigma_{2}^{(k)} \\
P_{1}^{(k n)} & P_{2}^{(k n)}
\end{array}\right|+i c L_{k n}\left|\begin{array}{ccc}
\sigma_{1}^{(k)} & \sigma_{2}^{(k)} \\
\xi_{1}^{(k n)} & \xi_{2}^{(k n)}
\end{array}\right|\right) .
\end{aligned}
$$

Now we are able to write in a vector form of the last three spin equations with radiation terms.

The last three equations can be written in a vector form

$$
\begin{aligned}
\frac{d\left(\vec{\lambda}^{(k)} \times \vec{\sigma}^{(k)}\right)}{d s_{k}}= & \sum_{n=1, n \neq k}^{3} \frac{e_{k} e_{n}}{m_{k} c^{2}}\left(\vec{P}^{(k n)}\left\langle\vec{\xi}^{(k n)} \times \vec{\lambda}^{(k)}, \vec{\sigma}^{(k)}\right\rangle-\vec{\xi}^{(k n)}\left\langle\vec{P}^{(k n)} \times \vec{\lambda}^{(k)}, \vec{\sigma}^{(k)}\right\rangle+\right. \\
& \left.+c^{2} \tau_{k n}\left(\vec{\sigma}^{(k)} \times \vec{P}^{(k n)}\right)+c^{2} L_{k n}\left(\vec{\sigma}^{(k)} \times \vec{\xi}^{(k n)}\right)\right)+ \\
& +\frac{e_{k}^{2}}{2 m_{k} c^{2}}\left\{\left(\vec{P}^{(k) r e t}\left\langle\vec{\xi}^{(k) r e t} \times \vec{\lambda}^{(k)}, \vec{\sigma}^{(k)}\right\rangle-\vec{\xi}^{(k) r e t}\left\langle\vec{P}^{(k) r e t} \times \vec{\lambda}^{(k)}, \vec{\sigma}^{(k)}\right\rangle+\right.\right. \\
& \left.+c^{2} \tau_{k}^{r e t}\left(\vec{\sigma}^{(k)} \times \vec{P}^{(k) r e t}\right)+c^{2} L_{k, r e t}\left(\vec{\sigma}^{(k)} \times \vec{\xi}^{(k) r e t}\right)\right)- \\
& -\left(\vec{P}^{(k) a d v}\left\langle\vec{\xi}^{(k) a d v} \times \vec{\lambda}^{(k)}, \vec{\sigma}^{(k)}\right\rangle-\vec{\xi}^{(k) a d v}\left\langle\vec{P}^{(k) a d v} \times \vec{\lambda}^{(k)}, \vec{\sigma}^{(k)}\right\rangle+\right. \\
& \left.\left.+c^{2} \tau_{k}^{a d v}\left(\vec{\sigma}^{(k)} \times \vec{P}^{(k) a d v}\right)+c^{2} L_{k, a d v}\left(\vec{\sigma}^{(k)} \times \vec{\xi}^{(k) a d v}\right)\right)\right\} .
\end{aligned}
$$

\section{The Last Three Equations are Consequences of the First Three Ones}

First, we rewrite the equations of motion in the form

$$
\begin{aligned}
\frac{d \vec{\lambda}^{(k)}}{d s_{k}} & =-\frac{e_{k}^{2}}{m_{k} c^{2}}\left[\vec{P}^{(k n)}\left\langle\lambda^{(k)}, \xi^{(k n)}\right\rangle_{4}-\vec{\xi}^{(k n)}\left\langle\lambda^{(k)}, P^{(k n)}\right\rangle_{4}\right] \\
\frac{d \vec{\lambda}^{(k)}}{d s_{k}} & =-\frac{e_{k}^{2}}{m_{k} c^{2}}\left[\vec{P}^{(k n)} \frac{\left\langle\vec{u}^{(k)}, \vec{\xi}^{(k n)}\right\rangle-c^{2} \tau_{k n}}{\Delta_{k}}-\vec{\xi}^{(k n)} \frac{\left\langle\vec{u}^{(k)}, \vec{P}^{(k n)}\right\rangle+c^{2} L_{k n}}{\Delta_{k}}\right]
\end{aligned}
$$

and

$$
\begin{aligned}
& \frac{d \vec{\sigma}^{(k)}}{d s_{k}}=\sum_{n=1, n \neq k}^{3} \frac{e_{k} e_{n}}{m_{k} c^{2}}\left[\vec{P}^{(k n)} \times\left(\vec{\sigma}^{(k)} \times \vec{\xi}^{(k n)}\right)+\tau_{k n} \vec{P}^{(k n)} \times\left(\vec{\lambda}^{(k)} \times \vec{\sigma}^{(k)}\right)-\vec{\xi}^{(k n)} \times\left(\vec{\sigma}^{(k)} \times \vec{P}^{(k n)}\right)+\right. \\
& \left.+L_{k n} \vec{\xi}^{(k n)} \times\left(\vec{\lambda}^{(k)} \times \vec{\sigma}^{(k)}\right)\right]+ \\
& +\frac{e_{k}{ }^{2}}{2 m_{k} c^{2}}\left\{\left[\vec{P}^{(k) r e t} \times\left(\vec{\sigma}^{(k)} \times \vec{\xi}^{(k) r e t}\right)+\tau_{k}^{r e t} \vec{P}^{(k) r e t} \times\left(\vec{\lambda}^{(k)} \times \vec{\sigma}^{(k)}\right)-\vec{\xi}^{(k) r e t} \times\left(\vec{\sigma}^{(k)} \times \vec{P}^{(k) r e t}\right)+\right.\right. \\
& \left.+L_{k, \text { ret }} \vec{\xi}^{(\vec{k}) r e t} \times\left(\vec{\lambda}^{(k)} \times \vec{\sigma}^{(k)}\right)\right]-\left[\vec{P}^{(k) a d v} \times\left(\vec{\sigma}^{(k)} \times \vec{\xi}^{(k) a d v}\right)+\tau_{k}^{a d v} \vec{P}^{(k) a d v} \times\left(\vec{\lambda}^{(k)} \times \vec{\sigma}^{(k)}\right)-\right. \\
& \left.\left.-\vec{\xi}^{(k) a d v} \times\left(\vec{\sigma}^{(k)} \times \vec{P}^{(k) a d v}\right)+L_{k, a d v} \vec{\xi}^{(k) a d v} \times\left(\vec{\lambda}^{(k)} \times \vec{\sigma}^{(k)}\right)\right]\right\} .
\end{aligned}
$$


Our main goal is to check that if the last two systems have a solution then possess a solution $\left(\vec{\lambda}^{(k)}, \vec{\sigma}^{(k)}\right)$, i.e.

$$
\begin{aligned}
& \frac{d\left(\vec{\lambda}^{(k)} \times \vec{\sigma}^{(k)}\right)}{d s_{k}}=\sum_{n=1, n \neq k}^{3} \frac{e_{k} e_{n}}{m_{k} c^{2}}\left(\vec{P} \vec{P}^{(k n)}\left|\begin{array}{ccc}
\xi_{1}^{(k n)} & \xi_{2}^{(k n)} & \xi_{3}^{(k n)} \\
\lambda_{1}^{(k)} & \lambda_{2}^{(k)} & \lambda_{3}^{(k)} \\
\sigma_{1}^{(k)} & \sigma_{2}^{(k)} & \sigma_{3}^{(k)}
\end{array}\right|-\vec{\xi}^{(k n)}\left|\begin{array}{ccc}
P_{1}^{(k n)} & P_{2}^{(k n)} & P_{3}^{(k n)} \\
\lambda_{1}^{(k)} & \lambda_{2}^{(k)} & \lambda_{3}^{(k)} \\
\sigma_{1}^{(k)} & \sigma_{2}^{(k)} & \sigma_{3}^{(k)}
\end{array}\right|+\right. \\
& \left.+c^{2} \tau_{k n}\left(\vec{\sigma}^{(k)} \times \vec{P}^{(k n)}\right)+c^{2} L_{k n}\left(\vec{\sigma}^{(k)} \times \vec{\xi}^{(k n)}\right)\right) \equiv \vec{R}_{2}
\end{aligned}
$$

has a solution too. We verify this implication just for the Lorentz part.

Indeed, multiplying 5.1 from the right by $\vec{\sigma}^{(k)}$ and 5.2 from the left by $\vec{\lambda}^{(k)}$ we get:

$$
\begin{aligned}
\frac{d \vec{\lambda}^{(k)}}{d s_{k}} \times \vec{\sigma}^{(k)} & =-\frac{e_{k}^{2}}{m_{k} c^{2}}\left[\left(\vec{P}^{(k n)} \times \vec{\sigma}^{(k)}\right) \frac{\left\langle\vec{u}^{(k)}, \vec{\xi}^{(k n)}\right\rangle-c^{2} \tau_{k n}}{\Delta_{k}}-\left(\vec{\xi}^{(k n)} \times \vec{\sigma}^{(k)}\right) \frac{\left\langle\vec{u}^{(k)}, \vec{P}^{(k n)}\right\rangle+c^{2} L_{k n}}{\Delta_{k}}\right] ; \\
\vec{\lambda}^{(k)} \times \frac{d \vec{\sigma}^{(k)}}{d s_{k}} & =\sum_{n=1, n \neq k}^{3} \frac{e_{k} e_{n}}{m_{k} c^{2}} \vec{\lambda}^{(k)} \times\left[\vec{P}^{(k n)} \times\left(\vec{\sigma}^{(k)} \times \vec{\xi}^{(k n)}\right)+\tau_{k n} \vec{P}^{(k n)} \times\left(\vec{\lambda}^{(k)} \times \vec{\sigma}^{(k)}\right)-\right. \\
& \left.-\vec{\xi}^{(k n)} \times\left(\vec{\sigma}^{(k)} \times \vec{P}^{(k n)}\right)+L_{k n} \vec{\xi}^{(k n)} \times\left(\vec{\lambda}^{(k)} \times \vec{\sigma}^{(k)}\right)\right] .
\end{aligned}
$$

We add the above last equations

$$
\begin{aligned}
& \frac{d \vec{\lambda}^{(k)}}{d s_{k}} \times \vec{\sigma}^{(k)}+\vec{\lambda}^{(k)} \times \frac{d \vec{\sigma}^{(k)}}{d s_{k}} \\
& =-\frac{e_{k}^{2}}{m_{k} c^{2}}\left[\left(\vec{P}^{(k n)} \times \vec{\sigma}^{(k)}\right) \frac{\left\langle\vec{u}^{(k)}, \vec{\xi}^{(k n)}\right\rangle-c^{2} \tau_{k n}}{\Delta_{k}}-\left(\vec{\xi}^{(k n)} \times \vec{\sigma}^{(k)}\right) \frac{\left\langle\vec{u}^{(k)}, \vec{P}^{(k n)}\right\rangle+c^{2} L_{k n}}{\Delta_{k}}\right]+ \\
& +\sum_{n=1, n \neq k}^{3} \frac{e_{k} e_{n} \vec{\lambda}^{(k)} \times\left[\vec{P}^{(k n)} \times\left(\vec{\sigma}^{(k)} \times \vec{\xi}^{(k n)}\right)+\tau_{k n} \vec{P}^{(k n)} \times\left(\vec{\lambda}^{(k)} \times \vec{\sigma}^{(k)}\right)-\right.}{\left.m^{(k n)} \times\left(\vec{\sigma}^{(k)} \times \vec{P}^{(k n)}\right)+L_{k n} \vec{\xi}^{(k n)} \times\left(\vec{\lambda}^{(k)} \times \vec{\sigma}^{(k)}\right)\right] \vec{R}_{1}}
\end{aligned}
$$

or

$$
\begin{gathered}
\frac{d \vec{\lambda}^{(k)}}{d s_{k}} \times \vec{\sigma}^{(k)}+\vec{\lambda}^{(k)} \times \frac{d \vec{\sigma}^{(k)}}{d s_{k}}=-\frac{e_{k}^{2}}{m_{k} c^{2}}\left[\left(\vec{P}^{(k n)} \times \vec{\sigma}^{(k)}\right) \frac{\left\langle\vec{u}^{(k)} \vec{\xi}^{(k n)}\right\rangle-c^{2} \tau_{k n}}{\Delta_{k}}-\left(\vec{\xi}^{(k n)} \times \vec{\sigma}^{(k)}\right) \frac{\left\langle\vec{u}^{(k)}, \vec{P}^{(k n)}\right\rangle+c^{2} L_{k n}}{\Delta_{k}}\right]+ \\
+\sum_{n=1, n \neq k}^{3} \frac{e_{k} e_{n}}{m_{k} c^{2}}\left[\left\langle\vec{\lambda}^{(k)}, \vec{\sigma}^{(k)} \times \vec{\xi}^{(k n)}\right\rangle \vec{P}^{(k n)}-\left\langle\vec{\lambda}^{(k)}, \vec{P}^{(k n)}\right\rangle\left(\vec{\sigma}^{(k)} \times \vec{\xi}^{(k n)}\right)-\tau_{k n}\left\langle\vec{\lambda}^{(k)}, \vec{P}^{(k n)}\right\rangle\left(\vec{\lambda}^{(k)} \times \vec{\sigma}^{(k)}\right)-\right. \\
\left.\quad-\left\langle\vec{\lambda}^{(k)}, \vec{\sigma}^{(k)} \times \vec{P}^{(k n)}\right\rangle \vec{\xi}^{(k n)}+\left\langle\vec{\lambda}^{(k)}, \vec{\xi}^{(k n)}\right\rangle\left(\vec{\sigma}^{(k)} \times \vec{P}^{(k n)}\right)-L_{k n}\left\langle\vec{\lambda}^{(k)}, \vec{\xi}^{(k n)}\right\rangle\left(\vec{\lambda}^{(k)} \times \vec{\sigma}^{(k)}\right)\right] .
\end{gathered}
$$

Denote by $\vec{R}_{1}(k)$ the right-hand side of the last equality and form the scalar product

$$
\left\langle\vec{R}_{1}^{(k)}, \vec{\sigma}^{(k)}\right\rangle=\sum_{n=1, n \neq k}^{3} \frac{e_{k} e_{n}}{m_{k} c^{2}}\left(\left\langle\vec{\lambda}^{(k)}, \vec{\sigma}^{(k)} \times \vec{\xi}^{(k n)}\right\rangle\left\langle\vec{P}^{(k n)}, \vec{\sigma}^{(k)}\right\rangle-\left\langle\vec{\lambda}^{(k)}, \vec{\sigma}^{(k)} \times \vec{P}^{(k n)}\right\rangle\left\langle\vec{\xi}^{(k n)}, \vec{\sigma}^{(k)}\right\rangle\right) .
$$

We have to show that the right-hand side $\vec{R}_{1}{ }^{(k)}$ of the last equation coincides (in weak sense) with the right-hand side $\vec{R}_{2}{ }^{(k)}$ of the following vector equation corresponding to the last three equations. For $\vec{R}_{2}{ }^{(k)}$ we have

$$
\left\langle\vec{R}_{2}{ }^{(k)}, \vec{\sigma}^{(k)}\right\rangle=\sum_{n=1, n \neq k}^{3} \frac{e_{k} e_{n}}{m_{k} c^{2}}\left(\left\langle\vec{P}^{(k n)}, \vec{\sigma}^{(k)}\right\rangle\left\langle\vec{\xi}^{(k n)} \times \vec{\lambda}^{(k)}, \vec{\sigma}^{(k)}\right\rangle-\left\langle\vec{\xi}^{(k n)}, \vec{\sigma}^{(k)}\right\rangle\left\langle\vec{P}^{(k n)} \times \vec{\lambda}^{(k)}, \vec{\sigma}^{(k)}\right\rangle\right) .
$$


Then we conclude that $\left\langle\vec{R}_{1}^{(k)}, \vec{\sigma}^{(k)}\right\rangle-\left\langle\vec{R}_{2}{ }^{(k)}, \vec{\sigma}^{(k)}\right\rangle=\left\langle\vec{R}_{1}{ }^{(k)}-\vec{R}_{2}{ }^{(k)}, \vec{\sigma}^{(k)}\right\rangle=0$. It is easy to see that the solutions of the last equation are $\vec{R}_{1}{ }^{(k)}-\vec{R}_{2}{ }^{(k)}=\overrightarrow{0}$ or $\vec{R}_{1}{ }^{(k)}-\vec{R}_{2}{ }^{(k)}=\vec{q} \times \frac{\vec{\sigma}^{(k)}}{\left\langle\vec{\sigma}^{(k)}, \vec{\sigma}^{(k)}\right\rangle}$, where $\vec{q} \mathrm{IS}$ an arbitrary chosen vector.

The transformation of the radiation parts can be accomplished in a similar way.

\section{Appendix}

(A1) We verify that $\sigma_{i j}{ }^{(k)} \lambda_{j}{ }^{(k)}=0$ (cf. [10]. [11]) $(i=1,2,3,4)$ in view of $\sigma_{i i}{ }^{(k)}=0$ :

$$
\begin{aligned}
& \sigma_{11}^{(p)} \lambda_{1}{ }^{(p)}+\sigma_{12}{ }^{(p)} \lambda_{2}{ }^{(p)}+\sigma_{13}{ }^{(p)} \lambda_{3}{ }^{(p)}+\sigma_{14}{ }^{(p)} \lambda_{4}{ }^{(p)}=\sigma_{12}^{(p)} \lambda_{2}{ }^{(p)}+\sigma_{13}^{(p)} \lambda_{3}{ }^{(p)}+\sigma_{14}^{(p)} \lambda_{4}{ }^{(p)}= \\
& =\sigma_{3}{ }^{(k)} \lambda_{2}{ }^{(k)}-\sigma_{2}{ }^{(k)} \lambda_{3}{ }^{(k)}+i \frac{\lambda_{2}^{(k)} \sigma_{3}^{(k)}-\lambda_{3}^{(k)} \sigma_{2}^{(k)}}{c} i c=-\left|\begin{array}{cc}
\sigma_{2}{ }^{(k)} & \sigma_{3}{ }^{(k)} \\
\lambda_{2}{ }^{(k)} & \lambda_{3}{ }^{(k)}
\end{array}\right|+\left|\begin{array}{cc}
\sigma_{2}{ }^{(k)} & \sigma_{3}{ }^{(k)} \\
\lambda_{2}{ }^{(k)} & \lambda_{3}{ }^{(k)}
\end{array}\right|=0 \text {; } \\
& \sigma_{21}{ }^{(k)} \lambda_{1}{ }^{(k)}+\sigma_{22}{ }^{(k)} \lambda_{2}{ }^{(k)}+\sigma_{23}{ }^{(k)} \lambda_{3}{ }^{(k)}+\sigma_{24}{ }^{(k)} \lambda_{4}{ }^{(k)}=\sigma_{21}^{(k)} \lambda_{1}{ }^{(k)}+\sigma_{23}^{(k)} \lambda_{3}{ }^{(k)}+\sigma_{24}^{(k)} \lambda_{4}{ }^{(k)}= \\
& =\sigma_{2}{ }^{(k)} \lambda_{1}{ }^{(k)}+\sigma_{1}{ }^{(k)} \lambda_{3}{ }^{(k)}+i \frac{\lambda_{3}^{(k)} \sigma_{1}^{(k)}-\lambda_{1}^{(k)} \sigma_{3}^{(k)}}{c} i c=\left|\begin{array}{cc}
\sigma_{1}{ }^{(k)} & \sigma_{3}{ }^{(k)} \\
\lambda_{1}{ }^{(k)} & \lambda_{3}{ }^{(k)}
\end{array}\right|-\left|\begin{array}{cc}
\sigma_{1}{ }^{(k)} & \sigma_{3}{ }^{(k)} \\
\lambda_{1}{ }^{(k)} & \lambda_{3}{ }^{(k)}
\end{array}\right|=0 ; \\
& \sigma_{31}{ }^{(k)} \lambda_{1}{ }^{(k)}+\sigma_{32}{ }^{(k)} \lambda_{2}{ }^{(k)}+\sigma_{33}{ }^{(k)} \lambda_{3}{ }^{(k)}+\sigma_{34}{ }^{(k)} \lambda_{4}{ }^{(k)}=\sigma_{31}^{(k)} \lambda_{1}{ }^{(k)}+\sigma_{32}^{(k)} \lambda_{2}{ }^{(k)}+\sigma_{34}^{(k)} \lambda_{4}{ }^{(k)}= \\
& =\sigma_{2}{ }^{(k)} \lambda_{1}{ }^{(k)}-\sigma_{1}{ }^{(k)} \lambda_{2}{ }^{(k)}+i \frac{\lambda_{1}^{(k)} \sigma_{2}^{(k)}-\lambda_{2}^{(k)} \sigma_{1}^{(k)}}{c} i c=-\left|\begin{array}{cc}
\sigma_{1}{ }^{(k)} & \sigma_{2}{ }^{(k)} \\
\lambda_{1}{ }^{(k)} & \lambda_{2}{ }^{(k)}
\end{array}\right|+\left|\begin{array}{cc}
\sigma_{1}{ }^{(k)} & \sigma_{2}{ }^{(k)} \\
\lambda_{1}{ }^{(k)} & \lambda_{2}{ }^{(k)}
\end{array}\right|=0 \text {; } \\
& \sigma_{41}{ }^{(k)} \lambda_{1}{ }^{(k)}+\sigma_{42}{ }^{(k)} \lambda_{2}{ }^{(k)}+\sigma_{43}{ }^{(k)} \lambda_{3}{ }^{(k)}+\sigma_{44}{ }^{(k)} \lambda_{4}{ }^{(k)}=\sigma_{41}{ }^{(k)} \lambda_{1}{ }^{(k)}+\sigma_{42}{ }^{(k)} \lambda_{2}{ }^{(k)}+\sigma_{43}{ }^{(k)} \lambda_{3}{ }^{(k)}= \\
& =-\frac{i\left(\lambda_{2}^{(k)} \sigma_{3}^{(k)}-\lambda_{3}^{(k)} \sigma_{2}^{(k)}\right) \lambda_{1}{ }^{(k)}}{c}-\frac{i\left(\lambda_{3}^{(k)} \sigma_{1}^{(k)}-\lambda_{1}^{(k)} \sigma_{3}^{(k)}\right) \lambda_{2}{ }^{(k)}}{c}-\frac{i\left(\lambda_{1}^{(k)} \sigma_{2}^{(k)}-\lambda_{2}^{(k)} \sigma_{1}^{(k)}\right) \lambda_{3}{ }^{(k)}}{c}= \\
& =\frac{i}{c}\left|\begin{array}{cc}
\sigma_{2}^{(k)} & \sigma_{3}^{(k)} \\
\lambda_{2}^{(k)} & \lambda_{3}^{(k)}
\end{array}\right| \lambda_{1}{ }^{(k)}-\frac{i}{c}\left|\begin{array}{cc}
\sigma_{1}^{(k)} & \sigma_{3}^{(k)} \\
\lambda_{1}^{(k)} & \lambda_{3}^{(k)}
\end{array}\right| \lambda_{2}{ }^{(k)}+\frac{i}{c}\left|\begin{array}{cc}
\sigma_{1}^{(k)} & \sigma_{2}^{(k)} \\
\lambda_{1}^{(k)} & \lambda_{2}^{(k)}
\end{array}\right| \lambda_{3}{ }^{(k)}=\frac{i}{c}\left|\begin{array}{ccc}
\lambda_{1}{ }^{(k)} & \lambda_{2}{ }^{(k)} & \lambda_{3}{ }^{(k)} \\
\sigma_{1}^{(k)} & \sigma_{2}^{(k)} & \sigma_{3}^{(k)} \\
\lambda_{1}{ }^{(k)} & \lambda_{2}^{(k)} & \lambda_{3}^{(k)}
\end{array}\right|=0 .
\end{aligned}
$$

(A2) Let us consider the equations

$$
\frac{d \sigma_{i j}^{(k)}}{d s_{k}}=\frac{e_{k}}{m_{k} c^{2}}\left(\left(\sum_{n=1, n \neq k}^{3} F_{i m}^{(k n)}+F_{i m}^{(k) r a d}\right) \sigma_{m j}^{(k)}-\sigma_{i m}^{(k)}\left(\sum_{n=1, n \neq k}^{3} F_{m j}^{(k n)}+F_{m j}^{(k) r a d}\right)\right) \equiv \frac{e_{k} \tilde{F}_{i j}^{(k)}}{m_{k} c^{2}} .
$$

We notice that $F_{i m}^{(k n)}=-F_{m i}^{(k n)} ; \quad F_{i m}^{(k) r a d}=-F_{m i}^{(k) r a d} ; \quad \sigma_{m j}^{(k)}=-\sigma_{j m}^{(k)}$.

Therefore

$$
\begin{gathered}
\tilde{F}_{i j}^{(k)}=\left(\sum_{n=1, n \neq k}^{3} F_{i m}^{(k n)}+F_{i m}^{(k) r a d}\right) \sigma_{m j}^{(k)}-\sigma_{i m}^{(k)}\left(\sum_{n=1, n \neq k}^{3} F_{m j}^{(k n)}+F_{m j}^{(k) r a d}\right)- \\
-\left(\left(\sum_{n=1, n \neq k}^{3} F_{m i}^{(k n)}+F_{m i}^{(k) r a d}\right) \sigma_{j m}^{(k)}-\sigma_{m i}^{(k)}\left(\sum_{n=1, n \neq k}^{3} F_{j m}^{(k n)}+F_{j m}^{(k) r a d}\right)\right)=-\tilde{F}_{j i}^{(k)} .
\end{gathered}
$$

It follows $\tilde{F}_{i i}^{(k)}=0(i=1,2.3,4)$. 


\section{References}

[1] V.G. Angelov, The N-body problem of classical electrodynamics" Physics Essays, vol. 6, No.2, 1993, $204-211$.

[2] V.G. Angelov, Two-body problem of classical electrodynamics with radiation terms - derivation of equations (I), International Journal of Theoretical and Mathematical Physics, vol. 5, 2015, 119-135.

[3] V.G. Angelov, Two-body problem of classical electrodynamics with radiation terms - periodic solutions (II), International Journal of Theoretical and Mathematical Physics, vol. 6, No.1, 2016, 1-25.

[4] V.G. Angelov, Spin two- body problem of classical electrodynamics with radiation terms (I) - derivation of spin equations, International Journal of Theoretical and Mathematical Physics, vol. 7, No.5, 2017, 132-154.

[5] V. G. Angelov, The electromagnetic three-body problem with radiation terms - derivation of equations of motion (I), Results in Nonlinear Analysis, vol. 3, No.2, 2020, 45-58.

[6] V.G. Angelov, The electromagnetic three-body problem with radiation terms - existence-uniqueness of periodic orbit (II), Results in Nonlinear Analysis, vol. 3, No.3, 2020, 137- 158.

[7] A.O. Barut, C. Onem, and N. Unal, The Classical Relativistic Two-Body Problem with Spin and Self-Interactions, Int. Centre for Theoretical Physics, Trieste, 1989, 1-19.

[8] G. Bauer, D.-A. Deckert, D. Durr, and G. Hinrichs, On irreversibility and radiation in classical electrodynamics of point particles, Journal of Statistical Physics, vol. 154, No.1-2: 610, 12 pages, 2014.

[9] G. Bauer, D.-A. Deckert, and D. Durr, On the existence of dynamics in Wheeler-Feynman electromagnetism, Journal of Applied Mathematics and Physics, vol. 64, No.4, 2012, 1087 -1124.

[10] H.C. Corben, and P. Stehle, Classical Mechanics. New York, London: J. Wiley and Sons, 1960.

[11] H.C. Corben, Spin in classical and quantum theory, Physical Review, vol. 121, No.6, 1961, 1833-1839.

[12] D.-A. Deckert, and G. Hinrichs, Electrodynamic two-body problem for prescribing initial date on the straight line, [Online], Available: http://arxiv.org/abs/1507.04991.

[13] D.-A. Deckert, D. Durr, and N. Vona, Delay equations of the Wheeler-Feynman type, in Proc. Sixth International Conference on Differential and Functional-Differential Equations (Moscow, August 14-21, 2011, Part 3, Journal of Contemporary Mathematics. Fundamental Directions, 47: 46, 2013, 13 pages.

[14] J.B. Hughes, A generalized Hamiltonian dynamics for relativistic particles with spin, Nuovo Cimento, vol. 20, No.2, 1961, 89-156.

[15] J. De Luca, Variational electrodynamics of atoms, Progress in Electromagnetism Research, B, 1-11, July 2013.

[16] M. Mathisson, Neue mechanik materieller systeme, Acta Physica Polonica, vol. 6, 1937, 163-227.

[17] P. Nyborg, Macroscopic motion of classical spinning particles, Nuovo Cimento, vol. 26, No.4, 1962, 821-830.

[18] A. Schild, and J.A. Schlosser, Fokker action principle for particles with charge, spin, and magnetic moment, J. Mathematical Physics, vol. 6, No.8, 1965, 1299-1306.

[19] R. Schiller, Quasi-classical theory of the spinning electron, Physical Review, vol. 125, No.3, 1962, $1116-1123$.

[20] J. Weyssenhoff, and A. Raabe, Relativistic dynamics of spin-fluids and spin-particles, Acta Physica Polonica, vol. 9, No.1, 1947, 7-18. 